\title{
N-Sulfonyloxy Carbamates as Re-oxidants for the Tethered Aminohydroxylation (TA) Reaction
}

\author{
Timothy J. Donohoe, Majid J. Chughtai, David J. Klauber, David Griffin \\ and Andrew D. Campbell
}

\section{Supplementary Material}

The compounds $1,{ }^{1} \mathbf{2},{ }^{2} 4,{ }^{2} \mathbf{6},{ }^{2} \mathbf{8},{ }^{3}$ and $\mathbf{1 0}{ }^{4}$ are already known in the literature.

\section{General experimental}

Tetrahydrofuran, acetonitrile and toluene were purified prior to use by filtration through two activated alumina columns (activated basic aluminium oxide, Brockmann I, standard grade, 150 mesh, $58 \AA$ ). Reagents obtained from Acros, Aldrich, Avocado, Fluka and Lancaster fine chemicals suppliers were used directly.

Flash column chromatography was carried out using silica gel $60(0.040-0.063 \mathrm{~mm})$ (Merck) using head pressure by means of head bellows. Thin layer chromatography was performed on commercially available pre-coated aluminium-backed plates $\left(0.25 \mathrm{~mm}\right.$ silica gel with fluorescent indicator $\left.\mathrm{UV}_{254}\right)$. Visualisation was achieved by either the quenching of UV fluorescence or $\mathrm{KMnO}_{4}$ stain.

${ }^{1} \mathrm{H}$ and ${ }^{13} \mathrm{C}$ NMR spectra were recorded on a Bruker AVANCE AV400 (400 MHz and 100.6 MHz), Bruker DPX400 (400 MHz and $100.6 \mathrm{MHz})$ or a Bruker AVANCE AV500 (500 MHz and 125.7 MHz) spectrometer. Signal positions were recorded in $\delta$ ppm with the abbreviations s, d, dd, ddd, t, dt, ddt, dtt, td, at, q, dq, ABq, quin., br and $\mathrm{m}$ denoting singlet, doublet, double doublet, double doublet of doublets, triplet, doublet of triplets, double doublet of tripets, double triplet of triplets, apparent triplet, triplet of doublets, quartet, doublet of quartets, $\mathrm{AB}$ quartet, quintet, broad and 
multiplet respectively. All NMR chemical shifts were referenced to residual solvent peaks or to $\mathrm{SiMe}_{4}$ as an internal standard. All coupling constants, $J$, are quoted in $\mathrm{Hz}$.

Infra-red spectra were recorded on a Bruker Tensor 27 FTIR spectrometer. Spectra were analysed either as thin films between $\mathrm{NaCl}$ plates, $\mathrm{KBr}$ disks or in a chloroform solution cell. Mass spectra $(\mathrm{m} / \mathrm{z})$ and HRMS were recorded under the conditions of electrospray (ES), chemical (CI) and field (FI) ionisation. Melting points were obtained using a Leica VMTG heated-stage microscope and are uncorrected.

"Petrol" refers to the fraction boiling in the range $40-60{ }^{\circ} \mathrm{C}$ unless otherwise stated and "ether" refers to diethyl ether.

\section{Procedure A: formation of $N$-hydroxy carbamates}

$N, N$ '-Carbonyl diimidazole (1.5 eq.) was added to a solution of alcohol (1.0 eq.) in acetonitrile $(5 \mathrm{~mL} / \mathrm{mmol}$ of substrate) and stirred, under argon, at room temperature until TLC indicated complete consumption of the alcohol (typically after $2 \mathrm{~h}$ ). Imidazole (4.0 eq.) and hydroxylamine hydrochloride (5.0 eq.) were added and stirring continued until TLC showed complete consumption of the adduct. After removal of the reaction solvent, the residue was partitioned between ethyl acetate and $1 \mathrm{M} \mathrm{HCl}$ followed by extraction of the aqueous phase with ethyl acetate. The organic phase was washed with brine, dried $\left(\mathrm{MgSO}_{4}\right)$, filtered and concentrated in vacuo to afford the crude product which was purified by flash column chromatography as indicated. 


\section{Procedure B: mesitylation of $N$-hydroxy carbamates}

To a solution of substrate $(1.0 \mathrm{mmol})$ in toluene:DMF $(4: 1,5 \mathrm{~mL} / \mathrm{mmol})$, cooled to $0{ }^{\circ} \mathrm{C}$, was added 2-mesitylenesulfonyl chloride (1.0 eq.) followed by dropwise addition of triethylamine (1.0 eq.). The reaction was maintained at $0{ }^{\circ} \mathrm{C}$ until TLC indicated complete consumption of starting material, then quenched by addition of $1 \mathrm{M} \mathrm{HCl}$ and extracted with ether. The organic phase was washed with brine, dried $\left(\mathrm{MgSO}_{4}\right)$, filtered and concentrated in vacuo to afford the crude product which was purified by flash column chromatography as indicated.

\section{Procedure C: Tethered aminohydroxylation of $N$-sulfonyloxy carbamates}

To a stirred solution of $N$-sulfonyloxy carbamate $(1.0$ eq. $)$ in ${ }^{n} \mathrm{PrOH}(12 \mathrm{~mL} / \mathrm{mmol})$ was added water $(12 \mathrm{~mL} / \mathrm{mmol})$ (all but $1 \mathrm{~mL}$ ) and $\mathrm{EtN}^{i} \operatorname{Pr}_{2}(0.05$ eq.). After $5 \mathrm{~min}$ stirring, a solution of $\mathrm{K}_{2} \mathrm{OsO}_{2}(\mathrm{OH})_{4}(0.04$ eq.) in water $(1 \mathrm{~mL})$ was slowly added and the reaction stirred. After TLC indicated that the reaction had ceased $\mathrm{Na}_{2} \mathrm{SO}_{3}$ (ca. $200 \mathrm{mg}$ ) was added, the reaction was stirred for $30 \mathrm{~min}$, then concentrated onto silica in vacuo and purified by flash column chromatography as indicated.

\section{$O$-2-Mesitylenesulfonyl $N$-hydroxy allyl carbamate 1}

Allyl chloroformate $(1.21 \mathrm{~g}, 10 \mathrm{mmol})$ was added to a stirred solution of hydroxylamine hydrochloride $(1.74 \mathrm{~g}, 25 \mathrm{mmol})$ and sodium hydroxide $(1.20 \mathrm{~g}$, $30 \mathrm{mmol})$ in water $(10 \mathrm{~mL})$ at room temperature. After $24 \mathrm{~h}$, the mixture was acidified with $c$. $\mathrm{HCl}$ then extracted with ether, dried $\left(\mathrm{MgSO}_{4}\right)$, filtered and concentrated in vacuo. The crude product was purified by flash column chromatography (petrol:ethyl acetate, 2:1) to furnish the $N$-hydroxy carbamate (675 mg, 58\%) as a clear oil. The N-hydroxy carbamate (673 mg, $5.75 \mathrm{mmol}$ ) was then subjected to the general mesitylation procedure and purified by flash column 
chromatography (petrol:ethyl acetate, 2:1) to afford $N$-sulfonyloxy carbamate 1 $(1.46 \mathrm{~g}, 85 \%)$ as a white solid; m.p. $96-98^{\circ} \mathrm{C} ; \delta_{\mathbf{H}}\left(400 \mathrm{MHz}, \mathrm{CDCl}_{3}\right) 7.81(1 \mathrm{H}, \mathrm{s})$, $7.00(2 \mathrm{H}, \mathrm{s}), 5.75(1 \mathrm{H}, \mathrm{ddt}, J 16.5,10.6,5.8), 5.23(1 \mathrm{H}, \mathrm{d}, J 5.1), 5.20(1 \mathrm{H}, \mathrm{s}), 4.51$ $(2 \mathrm{H}, \mathrm{d}, J 5.4), 2.68(6 \mathrm{H}, \mathrm{s}), 2.33(3 \mathrm{H}, \mathrm{s}) ; \delta_{\mathbf{C}}\left(100.6 \mathrm{MHz}, \mathrm{CDCl}_{3}\right)$ 155.2, 144.6, 142.0, $131.7,130.9,128.0,119.2,67.4,23.0,21.2$.

\section{(4RS)-Hydroxymethyl-oxazolidin-2-one 2}

$\mathrm{N}$-Sulfonyloxy carbamate $1(299 \mathrm{mg}, 1.00 \mathrm{mmol})$ was subjected to the general aminohydroxylation procedure. The crude product was purified by flash column chromatography (ethyl acetate) to furnish the title compound $(82.5 \mathrm{mg}, 70 \%)$ as a white solid; m.p. $78-83{ }^{\circ} \mathrm{C} ; \delta_{\mathbf{H}}\left(400 \mathrm{MHz}, \mathrm{D}_{2} \mathrm{O}\right) 4.46(1 \mathrm{H}, \mathrm{t}, J 9.0), 4.19(1 \mathrm{H}$, dd, $J 9.0,5.1), 3.98(1 \mathrm{H}, \mathrm{m}), 3.58(1 \mathrm{H}, \mathrm{dd}, J 12.0,3.7), 3.48(1 \mathrm{H}, \mathrm{dd}, J 12.0,4.3) ; \delta_{\mathrm{C}}$ (100.6 MHz, $\left.\mathrm{D}_{2} \mathrm{O}\right) 162.5,68.1,62.6,53.9$.

\section{$O$-2-Mesitylenesulfonyl $N$-hydroxy $(E)$-hex-2-enyl carbamate 3}

trans-Hex-2-enol $(1.00 \mathrm{~g}, 10.0 \mathrm{mmol})$ was subjected to the general $N$-hydroxy carbamate formation procedure and purified by flash column chromatography (petrol:ethyl acetate, $5: 1 \rightarrow 1: 1$ ) to afford the corresponding $\mathrm{N}$-hydroxy carbamate (1.56 g, 95\%). The N-hydroxy carbamate (959 mg, $6.02 \mathrm{~mol}$ ) was then subjected to the general mesitylation procedure and purified by flash column chromatography (petrol:ethyl acetate, 9:1) to afford the $\mathrm{N}$-sulfonyloxy carbamate $\mathbf{3}(1.82 \mathrm{~g}, 89 \%)$ as a white solid; m.p. $50-52{ }^{\circ} \mathrm{C} ; v_{\max }\left(\mathrm{CHCl}_{3}\right) 3281,1743,1373,1193 \mathrm{~cm}^{-1} ; \delta_{\mathbf{H}}(400 \mathrm{MHz}$, $\left.\mathrm{CDCl}_{3}\right) 7.86(1 \mathrm{H}, \mathrm{br}), 6.99(2 \mathrm{H}, \mathrm{s}), 5.70(1 \mathrm{H}, \mathrm{dt}, J 15.4,6.7), 5.38(1 \mathrm{H}, \mathrm{dtt}, J 15.4$, 6.6, 1.2), $4.45(2 \mathrm{H}, \mathrm{dd}, J$ 6.7, 0.9), $2.68(6 \mathrm{H}, \mathrm{s}), 2.33(3 \mathrm{H}, \mathrm{s}), 2.00(2 \mathrm{H}, 6.8), 1.39(2 \mathrm{H}$, sextet, J 7.3), $0.90\left(3 \mathrm{H}, \mathrm{t}, J\right.$ 7.3); $\delta_{\mathrm{C}}\left(100.6 \mathrm{MHz}, \mathrm{CDCl}_{3}\right) 155.4,144.5,142.0,137.7$, 
131.7, 128.3, 122.6, 67.7, 34.2, 23.0, 21.9, 21.2, 13.6; m/z $\left(\mathrm{ES}^{+}\right) 400.3\left(\mathrm{MNH}_{4}^{+}\right.$ MeCN, 100\%). HRMS (ES ${ }^{+}$Calcd. for $\mathrm{C}_{16} \mathrm{H}_{23} \mathrm{NO}_{5} \mathrm{SNa}\left(\mathrm{MNa}^{+}\right)$364.1189. Found 364.1182.

\section{(1'RS, 4RS)-4-[1'-Hydroxybutyl]-oxazolidin-2-one 4}

$\mathrm{N}$-Sulfonyloxy carbamate $\mathbf{3}(341 \mathrm{mg}, 1.00 \mathrm{mmol})$ was subjected to the general aminohydroxylation procedure. The crude material was purified by flash column chromatography (ethyl acetate) to furnish the title compound as a white solid $(117 \mathrm{mg}, 74 \%)$; m.p. $71-73{ }^{\circ} \mathrm{C} ; \delta_{\mathbf{H}}\left(400 \mathrm{MHz}, \mathrm{CDCl}_{3}\right) 6.49(1 \mathrm{H}, \mathrm{br}), 4.48(1 \mathrm{H}, \mathrm{t}$, $J$ 8.5), $4.23(1 \mathrm{H}, \mathrm{dd}, J$ 8.5, 5.5), $3.81(1 \mathrm{H}, \mathrm{dt}, J 9.0,5.5),, 3.58-3.48(2 \mathrm{H}, \mathrm{m}), 1.59$ $(1 \mathrm{H}, \mathrm{m}), 1.49-1.30(3 \mathrm{H}, \mathrm{m}), 0.98(3 \mathrm{H}, \mathrm{t}, J 7.0) ; \delta_{\mathrm{C}}\left(100.6 \mathrm{MHz}, \mathrm{CDCl}_{3}\right) 160.8,72.7$ $67.4,57.4,35.2,18.7,13.9$.

\section{$O$-2-Mesitylenesulfonyl $N$-hydroxy $(Z)$-hex-2-enyl carbamate 5}

cis-Hex-2-enol (501 mg, $5.00 \mathrm{mmol})$ was subjected to the general $N$-hydroxy carbamate formation procedure and purified by flash column chromatography (petrol:ethyl acetate, $3: 1 \rightarrow 1: 1$ ) to afford the corresponding $\mathrm{N}$-hydroxy carbamate (751 mg, 94\%). The N-hydroxy carbamate (681 mg, $4.28 \mathrm{mmol}$ ) was then subjected to the general mesitylation procedure and purified by flash column chromatography (petrol:ethyl acetate, $9: 1 \rightarrow 1: 1)$ to afford $\mathrm{N}$-sulfonyloxy carbamate $7(1.12 \mathrm{~g}, 77 \%)$ as

a white solid; m.p. $35-37{ }^{\circ} \mathrm{C}$; $v_{\max }$ (thin film) $3282,1742,1373,1193 \mathrm{~cm}^{-1} ; \delta_{\mathbf{H}}$ $\left(400 \mathrm{MHz}, \mathrm{CDCl}_{3}\right) 8.56(1 \mathrm{H}, \mathrm{s}), 7.52(2 \mathrm{H}, \mathrm{s}), 6.17(1 \mathrm{H}, \mathrm{dt}, J 11.0,7.5), 5.89(1 \mathrm{H}, \mathrm{dtt}$ $J$ 11.0, 7.1, 1.4), 5.09 (2H, dd, J 7.1, 1.4), $3.21(6 \mathrm{H}, \mathrm{s}), 2.86(3 \mathrm{H}, \mathrm{s}), 2.53(2 \mathrm{H}, \mathrm{dq}$, $J 7.5,1.1), 1.90(1 \mathrm{H}$, sextet, $J 7.5), 1.42(3 \mathrm{H}, \mathrm{t}, J 7.5) ; \delta_{\mathrm{C}}\left(100.6 \mathrm{MHz}, \mathrm{CDCl}_{3}\right)$ 155.6, $144.5,142.0,136.2,131.7,128.2,122.2,62.7,28.4,23.0,22.5,21.2,13.6 ; m / z\left(\mathrm{ES}^{+}\right)$ 
$364.2\left(\mathrm{MNa}^{+}, 35\right), 400.3\left(\mathrm{MNH}_{4}{ }^{+} \mathrm{MeCN}, \quad 70 \%\right)$. HRMS $\left(\mathrm{ES}^{+}\right)$Calcd. for $\mathrm{C}_{16} \mathrm{H}_{23} \mathrm{NNaO}_{5} \mathrm{~S}\left(\mathrm{MNa}^{+}\right)$364.1189. Found 364.1185.

\section{(1'SR, 4RS)-4-[1'-Hydroxybutyl]-oxazolidin-2-one 6}

$\mathrm{N}$-Sulfonyloxy carbamate $5(341 \mathrm{mg}, 1.00 \mathrm{mmol})$ was subjected to the general aminohydroxylation procedure. The crude material was purified by flash column chromatography (ethyl acetate) to furnish the title compound (132 $\mathrm{mg}, 83 \%)$ as a white solid; m.p. $72-74{ }^{\circ} \mathrm{C} ; \delta_{\mathbf{H}}\left(400 \mathrm{MHz}, \mathrm{CDCl}_{3}\right) 6.45(1 \mathrm{H}, \mathrm{br}), 4.47(1 \mathrm{H}, \mathrm{dd}, J$ 8.5, 5.5), $4.42(1 \mathrm{H}, \mathrm{t}, J 8.5), 3.87(1 \mathrm{H}, \mathrm{m}), 3.74(1 \mathrm{H}, \mathrm{m}), 2.70(1 \mathrm{H}, \mathrm{br}), 1.60-1.54(1 \mathrm{H}, \mathrm{m})$, 1.44-1.29 (3H, m), $0.97(3 \mathrm{H}, \mathrm{m},) ; \delta_{\mathrm{C}}\left(100.6 \mathrm{MHz}, \mathrm{CDCl}_{3}\right)$ 160.9, 71.0, 65.8, 56.9, $34.3,18.9,13.9$.

\section{$O$-2-Mesitylenesulfonyl $N$-hydroxy cyclohex-2-enyl carbamate 7}

Cyclohex-2-enol $(980 \mathrm{mg}, 10.0 \mathrm{mmol})$ was subjected to the general $N$-hydroxy carbamate formation procedure and purified by flash column chromatography (petrol:ether, 3:1) to afford the corresponding $\mathrm{N}$-hydroxy carbamate (1.10 g, 70\%). The N-hydroxy carbamate $(490 \mathrm{mg}, 3.12 \mathrm{mmol}$ ) was then subjected to the general mesitylation procedure and purified by flash column chromatography (petrol:ether, 4:1) to afford N-sulfonyloxy carbamate $7(950 \mathrm{mg}, 90 \%)$ as a white solid; m.p. decomposed at $95^{\circ} \mathrm{C} ; v_{\max }\left(\mathrm{CHCl}_{3}\right) 3362,1728,1374,1194 \mathrm{~cm}^{-1} ; \delta_{\mathbf{H}}(400 \mathrm{MHz}$, $\left.\mathrm{CDCl}_{3}\right) 7.76(1 \mathrm{H}, \mathrm{s}), 6.99(2 \mathrm{H}, \mathrm{s}), 5.95(1 \mathrm{H}, \mathrm{dt}, J 5.9,3.2), 5.52(1 \mathrm{H}, \mathrm{dt}, J$ 10.0, 1.7), $5.09(1 \mathrm{H}, \mathrm{m}), 2.68(6 \mathrm{H}, \mathrm{s}), 2.33(3 \mathrm{H}, \mathrm{s}), 2.09-1.93(2 \mathrm{H}, \mathrm{m}), 1.79-1.66(4 \mathrm{H}, \mathrm{m}) ; \delta_{\mathrm{C}}$ $\left(100.6 \mathrm{MHz}, \mathrm{CDCl}_{3}\right)$ 155.3, 144.5, 142.0, 133.6, 131.7, 128.4, 124.4, 71.4, 27.9, 24.7, 23.0, 21.2, 18.4; $\boldsymbol{m} / \boldsymbol{z}\left(\mathrm{ES}^{+}\right) 363.3\left(\mathrm{MNa}^{+}, 5\right), 398.3\left(\mathrm{MNH}_{4}{ }^{+} \mathrm{MeCN}, 50 \%\right)$. HRMS $\left(\mathrm{ES}^{+}\right)$Calcd. for $\mathrm{C}_{16} \mathrm{H}_{21} \mathrm{NO}_{5} \mathrm{NaS}\left(\mathrm{MNa}^{+}\right)$362.1038. Found 362.1046. 


\section{(3SR, 4SR, 8RS)-4-Hydroxy-hexahydro-benzooxazol-2-one 8}

$N$-Sulfonyloxy carbamate $7(170 \mathrm{mg}, 0.50 \mathrm{mmol})$ was subjected to the general aminohydroxylation procedure. The crude material was purified by flash column chromatography (ethyl acetate) to furnish the title compound $(62.8 \mathrm{mg}, 80 \%)$ as a white solid; m.p. $80-81^{\circ} \mathrm{C}$; $\delta_{\mathbf{H}}\left(400 \mathrm{MHz}, \mathrm{CDCl}_{3}\right) 6.60(1 \mathrm{H}, \mathrm{s}), 4.69(1 \mathrm{H}, \mathrm{dt}, J$ 6.6, 5.1), 4.10-3.70 (1H, br), 3.95 (1H, dd, $J 8.1,4.3), 3.87$ (1H, dt, $J 8.5,4.4), 1.79-1.62$ $(5 \mathrm{H}, \mathrm{m}), 1.38-1.33(1 \mathrm{H}, \mathrm{m}) ; \delta_{\mathrm{C}}\left(100.6 \mathrm{MHz}, \mathrm{CDCl}_{3}\right)$ 161.5, 76.4, 66.8, 55.8, 26.7, $25.8,15.6$.

\section{$O$-2-Mesitylenesulfonyl $N$-hydroxy 1-cyclohexene-1-yl-pentyl carbamate 9}

${ }^{\mathrm{n}} \mathrm{BuLi}$ (1.6 M, $12.4 \mathrm{~mL}, 20.0 \mathrm{mmol}$ ) was added dropwise to a stirred solution of 1cyclohexene-1-carboxaldehyde $(2.00 \mathrm{~g}, 18.0 \mathrm{mmol})$ in THF $(50 \mathrm{~mL})$ at $-78^{\circ} \mathrm{C}$ under argon. The solution was allowed to stir at $-78^{\circ} \mathrm{C}$ for 30 minutes before being allowed to warm to room temperature. The reaction was quenched by the addition of water $(20 \mathrm{~mL})$ and diluted with ether. The mixture was washed with aqueous $\mathrm{HCl}$ $(2 \mathrm{M})$ and brine. The organic layer was collected, dried $\left(\mathrm{MgSO}_{4}\right)$, filtered and concentrated in vacuo to afford the alcohol $(2.60 \mathrm{~g}, 86 \%)$ without purification as an oil. The alcohol $(776 \mathrm{mg}, 4.61 \mathrm{mmol})$ was subjected to the general $N$-hydroxy carbamate formation procedure and purified by flash column chromatography (petrol:ethyl acetate, $4: 1 \rightarrow 2: 1$ ) to afford the corresponding $\mathrm{N}$-hydroxy carbamate (519 $\mathrm{mg}, 49 \%)$. The $N$-hydroxy carbamate $(227 \mathrm{mg}, 1.00 \mathrm{mmol})$ was then subjected to the general mesitylation procedure and purified by flash column chromatography (petrol:acetone, $97: 3 \rightarrow 90: 10)$ to afford $\mathrm{N}$-sulfonyloxy carbamate $\mathbf{9}(322 \mathrm{mg}, 79 \%)$ as a clear oil; $v_{\max }\left(\right.$ thin film) 3362, 1728, 1374, $1194 \mathrm{~cm}^{-1} ; \delta_{\mathbf{H}}\left(400 \mathrm{MHz} ; \mathrm{CDCl}_{3}\right) 7.89$ $(1 \mathrm{H}, \mathrm{s}), 6.98(2 \mathrm{H}, \mathrm{s}), 5.53(1 \mathrm{H}, \mathrm{m}), 4.91(1 \mathrm{H}$, at, $J 7.0), 2.68(6 \mathrm{H}, \mathrm{s}), 2.32(3 \mathrm{H}, \mathrm{s})$, 
2.01-1.90 (2H, m), 1.78-1.76 (2H, m), 1.62-1.44 (6H, m), 1.29-1.20 (2H, m), 1.11$1.04(2 \mathrm{H}, \mathrm{m}), 0.86\left(3 \mathrm{H}, \mathrm{t}, J\right.$ 7.4); $\delta_{\mathbf{C}}\left(100.6 \mathrm{MHz} ; \mathrm{CDCl}_{3}\right)$ 155.2, 144.4, 142.0, 141.9, $134.8,131.7,131.6,128.4,126.0,82.0,31.9,27.4,24.8,23.4,23.0,22.3,21.8,21.2$ 21.1, 13.9; $\boldsymbol{m} / \boldsymbol{z}\left(\mathrm{ES}^{+}\right) 432.3\left(\mathrm{MNa}^{+}, 15\right), 468.4\left(\mathrm{MNH}_{4}{ }^{+} \mathrm{MeCN}, 100 \%\right) . \mathbf{H R M S}\left(\mathrm{ES}^{+}\right)$ Calcd. for $\mathrm{C}_{21} \mathrm{H}_{31} \mathrm{NO}_{5} \mathrm{SNa}\left(\mathrm{MNa}^{+}\right)$432.1815. Found 432.1805.

\section{(4RS, 5SR, 6SR)-4-Butyl-6-hydroxy-3oxa-1-aza-spiro[4.5]decan-2-one 10}

$N$-Sulfonyloxy carbamate $9(225 \mathrm{mg}, 0.55 \mathrm{mmol})$ was subjected to the general aminohydroxylation procedure. The crude material was purified by flash column chromatography (ethyl acetate:petrol, 2:1) to furnish the title compound (84.0 mg, $67 \%, 20: 1)$ as a clear oil; $\delta_{\mathbf{H}}\left(400 \mathrm{MHz} ; \mathrm{CDCl}_{3}\right)$ (major isomer) $7.32(1 \mathrm{H}, \mathrm{br}), 4.69$ $(1 \mathrm{H}, \mathrm{dd}, J 7.0,2.8), 3.28(1 \mathrm{H}, \mathrm{dd}, J 10.4,4.3), 2.53(1 \mathrm{H}, \mathrm{br}), 1.90(1 \mathrm{H}, \mathrm{m}), 1.82(1 \mathrm{H}$ m), $1.72(1 \mathrm{H}, \mathrm{m}), 1.63-1.20(11 \mathrm{H}, \mathrm{m}), 0.90\left(3 \mathrm{H}, \mathrm{t}, J\right.$ 7.4); $\delta_{\mathrm{C}}\left(100.6 \mathrm{MHz} ; \mathrm{CDCl}_{3}\right)$ $160.3,81.8,71.8,64.3,60.4,31.7,30.7,29.2,28.3,23.7,21.2,13.9$.

\section{$O$-2-Mesitylenesulfonyl $N$-hydroxy $(E)$-hex-3-enyl carbamate 11}

trans-Hex-3-enol $(500 \mathrm{mg}, 5.00 \mathrm{mmol})$ was subjected to the general $N$-hydroxy carbamate formation procedure and purified by flash column chromatography (petrol:ether, 4:1) to afford the corresponding N-hydroxy carbamate (557 mg, 71\%). The $N$-hydroxy carbamate $(190 \mathrm{mg}, 1.19 \mathrm{mmol})$ was then subjected to the general mesitylation procedure and purified by flash column chromatography (petrol:ether, 5:1) to afford N-sulfonyloxy carbamate 11 (317 mg, 78\%) as a white solid; m.p. 59$61{ }^{\circ} \mathrm{C} ; v_{\max }\left(\mathrm{KBr}\right.$ disk) 3281, 1744, 1603, 1373, $1193 \mathrm{~cm}^{-1} ; \delta_{\mathbf{H}}\left(400 \mathrm{MHz}, \mathrm{CDCl}_{3}\right)$ $7.78(1 \mathrm{H}, \mathrm{br}), 7.00(2 \mathrm{H}, \mathrm{s}), 5.50(1 \mathrm{H}, \mathrm{dt}, J 15.3,6.3), 5.24$ (1H. dt, $J 15.4,6.7), 4.02$ (2H, t, J 6.9), $2.69(6 \mathrm{H}, \mathrm{s}), 2.33(3 \mathrm{H}, \mathrm{s}), 2.20(2 \mathrm{H}, \mathrm{q}, J$ 6.5), 1.99 (2H, quin, $J$ 7.4), 
$0.96\left(3 \mathrm{H}, \mathrm{t}, J\right.$ 7.5); $\delta_{\mathrm{C}}\left(100.6 \mathrm{MHz}, \mathrm{CDCl}_{3}\right) 155.5,144.6,142.0,135.7,131.7,128.1$ 123.0, 66.7, 31.7, 25.6, 23.0, 21.2, 13.6; m/z $\left(\mathrm{ES}^{+}\right) 364.0\left(\mathrm{MNa}^{+}, 100\right), 400.2$ $\left(\mathrm{MNH}_{4}{ }^{+} \mathrm{MeCN}, \quad 55\right) \quad 405.2 \quad\left(\mathrm{MNa}^{+} \mathrm{MeCN}, \quad 50 \%\right) . \quad$ HRMS $\left(\mathrm{ES}^{+}\right)$Calcd. for $\mathrm{C}_{16} \mathrm{H}_{23} \mathrm{NO}_{5} \mathrm{SNa}\left(\mathrm{MNa}^{+}\right)$364.1189. Found 364.1184.

\section{(4RS,1'RS)-4-(1'-Hydroxypropyl)-[1,3]oxazinan-2-one 12}

$\mathrm{N}$-Sulfonyloxy carbamate $11(171 \mathrm{mg}, 0.50 \mathrm{mmol})$ was subjected to the general aminohydroxylation procedure. The crude material was purified by flash column chromatography (ethyl acetate) to furnish the title compound $(50.0 \mathrm{mg}, 63 \%)$ as a pale yellow oil; $v_{\max }\left(\right.$ thin film) 3331, 3241, $1729,1656 \mathrm{~cm}^{-1} ; \delta_{\mathbf{H}}\left(400 \mathrm{MHz} ; \mathrm{CDCl}_{3}\right) 6.49$ (1H, br), $4.39-4.33(1 \mathrm{H}, \mathrm{m}), 4.17(1 \mathrm{H}, \mathrm{td}, J 11.4,2.5), 3.92(1 \mathrm{H}, \mathrm{br}), 3.36-3.29$ $(2 \mathrm{H}, \mathrm{m}), 1.97-1.92(1 \mathrm{H}, \mathrm{m}), 1.77-1.64(1 \mathrm{H}, \mathrm{m}), 1.62-1.52(1 \mathrm{H}, \mathrm{m}), 1.40(1 \mathrm{H}$ sextet, $J$ 7.5), $1.00\left(3 \mathrm{H}, \mathrm{t}, J\right.$ 7.5); $\delta_{\mathrm{C}}\left(100.6 \mathrm{MHz} ; \mathrm{CDCl}_{3}\right)$ 155.0, 75.6, 55.3, 65.6, 26.1, 24.3, 9.5; $\boldsymbol{m} / \boldsymbol{z}(\mathrm{CI}) 177\left(6 \%, \mathrm{M}+\mathrm{NH}_{4}{ }^{+}\right), 160\left(100 \%, \mathrm{M}+\mathrm{H}^{+}\right), 142(30 \%, \mathrm{M}-$ $\mathrm{OH})$; HRMS (CI) Calcd. for $\mathrm{C}_{7} \mathrm{H}_{14} \mathrm{NO}_{3}\left(\mathrm{MH}^{+}\right)$160.0974. Found 160.0967.

\section{Osmate Ester 13}

TMEDA $(57.5 \mu \mathrm{L}, 0.38 \mathrm{mmol})$ was added to a solution of $\mathrm{N}$-sulfonyloxy carbamate 11 in ${ }^{n} \mathrm{PrOH}(8 \mathrm{~mL})$ and stirred for $5 \mathrm{~min} . \mathrm{K}_{2} \mathrm{OsO}_{2}(\mathrm{OH})_{4}(141 \mathrm{mg}, 0.38 \mathrm{mmol})$ in water $(0.50 \mathrm{~mL})$ was added with stirring with the reaction turning brown. After $24 \mathrm{~h}$, the reaction was diluted with water $(10 \mathrm{~mL})$ and extracted with dichloromethane $(3 \times$ $10 \mathrm{~mL})$. The combined organic phase was dried $\left(\mathrm{MgSO}_{4}\right)$, filtered and concentrated in vacuo to give an orange/brown solid which was purified by flash column chromatography (dichloromethane:methanol, 19:1) to afford osmate ester 13 (67 mg, $36 \%$ ) as an orange-brown solid; m.p. (dec. $\left.180^{\circ} \mathrm{C}\right) ; v_{\max }\left(\mathrm{KBr}\right.$ disk) $1657 \mathrm{~cm}^{-1} ; \delta_{\mathrm{H}}$ 
(400 MHz, $\left.\mathrm{CDCl}_{3}\right)$ 4.36-4.32 (1H, m), $4.27(1 \mathrm{H}, \mathrm{td}, J 10.7,1.3), 3.88(1 \mathrm{H}, \mathrm{td}, J$ 8.4, 3.0), $3.61(1 \mathrm{H}, \mathrm{td}, J 9.6,4.5), 3.32(2 \mathrm{H}, \mathrm{q}, J 11.5), 3.10(3 \mathrm{H}, \mathrm{s}), 2.97(3 \mathrm{H}, \mathrm{s}), 2.94$ $(3 \mathrm{H}, \mathrm{s}), 2.87-2.82(2 \mathrm{H}, \mathrm{m}), 2.73(3 \mathrm{H}, \mathrm{s}), 2.65-2.62(1 \mathrm{H}, \mathrm{m}), 2.00-1.81(2 \mathrm{H}, \mathrm{m}), 1.59$ $(1 \mathrm{H}, \mathrm{m}), 1.07\left(3 \mathrm{H}, \mathrm{t}, J\right.$ 7.4); $\delta_{\mathrm{C}}\left(100.6 \mathrm{MHz}, \mathrm{CDCl}_{3}\right)$ 158.9, 94.8, 70.8, 65.5, 64.8, 62.9, 53.4, 52.1, 49.7, 47.1, 25.7, 25.6, 9.8; m/z $\left(\mathrm{ES}^{+}\right) 298.2\left(\mathrm{MH}^{+}, 45\right), 556.2$ $\left(\mathrm{MNH}_{4}{ }^{+} \mathrm{MeCN}, \quad 100 \%\right)$ HRMS $\left(\mathrm{ES}^{+}\right)$Calcd. for $\mathrm{C}_{13} \mathrm{H}_{27} \mathrm{~N}_{3} \mathrm{NaO}_{5} \mathrm{Os}\left(\mathrm{MNa}^{+}\right)$ 520.1458. Found 520.1462.

\section{$O$-2-Mesitylenesulfonyl $N$-hydroxy but-3-enyl carbamate 14}

But-2-en-1-ol (1.44 g, $20.0 \mathrm{mmol})$ was subjected to the general $N$-hydroxy carbamate formation procedure and purified by flash column chromatography (petrol:ethyl acetate, 1:1) to afford the corresponding $\mathrm{N}$-hydroxy carbamate $(3.49 \mathrm{~g}, 75 \%)$. The $\mathrm{N}$ Hydroxy carbamate $(1.51 \mathrm{~g}, 11.6 \mathrm{~mol})$ was then subjected to the general mesitylation procedure and purified by flash column chromatography (dichloromethane) to afford the N-sulfonyloxy carbamate $14(3.20 \mathrm{~g}, 88 \%)$ as a white solid; m.p. $69-70{ }^{\circ} \mathrm{C} ; \mathrm{v}_{\max }$ $\left(\mathrm{CHCl}_{3}\right) 3281,1745,1372,1193 \mathrm{~cm}^{-1} ; \delta_{\mathbf{H}}\left(400 \mathrm{MHz}, \mathrm{CDCl}_{3}\right) 7.79(1 \mathrm{H}, \mathrm{br}), 7.00(2 \mathrm{H}$ s), $5.65(1 \mathrm{H}, \mathrm{m}), 5.05(2 \mathrm{H}, \mathrm{m}), 4.08(2 \mathrm{H}, \mathrm{dt}, J$ 6.8, 1.0), $2.69(6 \mathrm{H}, \mathrm{s}), 2.33(3 \mathrm{H}, \mathrm{s})$, $2.28(2 \mathrm{H}, \mathrm{dq}, J 6.8,1.0) ; \delta_{\mathrm{C}}\left(100.6 \mathrm{MHz}, \mathrm{CDCl}_{3}\right) 155.4,144.6,142.0,133.0,131.7$ 128.3, 117.8, 66.0, 32.8, 23.0, 21.2; $\mathbf{m} / z\left(\mathrm{ES}^{+}\right) 336.0\left(\mathrm{MNa}^{+}, 30\right), 372.1\left(\mathrm{MNH}_{4}^{+}\right.$ MeCN, 100\%). HRMS (ES ${ }^{+}$Calcd. for $\mathrm{C}_{14} \mathrm{H}_{19} \mathrm{NO}_{5} \mathrm{SNa}\left(\mathrm{MNa}^{+}\right)$336.0876. Found 336.0888 .

\section{4-(Hydroxymethyl)-1,3-oxazinan-2-one 15}

$\mathrm{N}$-Sulfonyloxy carbamate $14(313 \mathrm{mg}, 1.00 \mathrm{mmol})$ was subjected to the general aminohydroxylation procedure. The crude material was purified by flash column 
chromatography (acetone:petrol, 2:1) to furnish the title compound (77.3 $\mathrm{mg}, 59 \%)$ as a white solid; m.p. $89-91^{\circ} \mathrm{C} ; v_{\max }\left(\mathrm{CHCl}_{3}\right) 3244,1711 \mathrm{~cm}^{-1} ; \delta_{\mathbf{H}}\left(400 \mathrm{MHz}, \mathrm{CDCl}_{3}\right)$ $6.50(1 \mathrm{H}, \mathrm{br}), 4.38(1 \mathrm{H}, \mathrm{dt}, J 11.1,4.2), 4.25(1 \mathrm{H}, \mathrm{td}, J 11.1,2.9), 3.74$ (1H, dd, $J 9.0$, 3.5), $3.65(1 \mathrm{H}, \mathrm{m}), 3.52(1 \mathrm{H}, \mathrm{dd}, J 9.0,7.5), 2.41(1 \mathrm{H}, \mathrm{br}), 1.98-1.92(1 \mathrm{H}, \mathrm{m}), 1.84-$ $1.74(1 \mathrm{H}, \mathrm{m}) ; \delta_{\mathrm{C}}\left(100.6 \mathrm{MHz}, \mathrm{CDCl}_{3}\right)$ 155.2, 65.5, 65.4, 52.4, 23.4. m/z $\left(\mathrm{ES}^{+}\right) 189.9$ $\left(\mathrm{MNH}_{4}{ }^{+} \mathrm{MeCN}, 10\right), 194.8\left(\mathrm{MNa}^{+} \mathrm{MeCN}, 15 \%\right)$. HRMS $\left(\mathrm{ES}^{+}\right)$Calcd. for $\mathrm{C}_{5} \mathrm{H}_{9} \mathrm{NO}_{3} \mathrm{Na}\left(\mathrm{MNa}^{+}\right)$154.0475. Found 154.0468. Elemental analysis Found: $\mathrm{C}, 45.5$ $\mathrm{H}, 6.93 ; \mathrm{N}, 10.4 . \mathrm{C}_{5} \mathrm{H}_{9} \mathrm{NO}_{3}$ requires $\mathrm{C}, 45.8 ; \mathrm{H}, 6.92 ; \mathrm{N}, 10.7$.

\section{$O$-2-Mesitylenesulfonyl $N$-hydroxy $(Z)$-hex-3-enyl carbamate 16}

cis-Hex-3-enol (500 mg, $5.00 \mathrm{mmol})$ was subjected to the general $N$-hydroxy carbamate formation procedure and purified by flash column chromatography (petrol:ethyl acetate, 3:1) to afford the corresponding N-hydroxy carbamate (540 mg, 68\%). The N-hydroxy carbamate $(515 \mathrm{mg}, 3.42 \mathrm{mmol}$ ) was then subjected to the general mesitylation procedure and purified by flash column chromatography (petrol:dichloromethane, 1:1) to afford $\mathrm{N}$-sulfonyloxy carbamate 16 (866 mg, 78\%) as a clear oil; $v_{\max }\left(\mathrm{CHCl}_{3}\right) 3281,1744,1373,1193 \mathrm{~cm}^{-1} ; \delta_{\mathbf{H}}\left(400 \mathrm{MHz}, \mathrm{CDCl}_{3}\right) 7.92$ $(1 \mathrm{H}, \mathrm{s}), 7.00(2 \mathrm{H}, \mathrm{s}), 5.48(1 \mathrm{H}, \mathrm{dt}, J 10.5,7.4), 5.17(1 \mathrm{H}, \mathrm{dt}, J 10.5,7.3), 4.01(2 \mathrm{H}, \mathrm{at}$, $J$ 7.0), $2.68(6 \mathrm{H}, \mathrm{s}), 2.33(3 \mathrm{H}, \mathrm{s}), 2.27(2 \mathrm{H}, \mathrm{q}, J 7.2), 2.00(2 \mathrm{H}$, quin, $J 7.6), 0.96(3 \mathrm{H}$, $\mathrm{t}, J$ 7.4); $\delta_{\mathrm{C}}\left(100.6 \mathrm{MHz}, \mathrm{CDCl}_{3}\right) 155.6,144.6,141.2,135.1,131.7,128.3,122.6$ 66.4, 26.5, 23.0, 21.2, 20.6, 14.2; $\mathbf{m} / z\left(\mathrm{ES}^{+}\right) 359.3\left(\mathrm{MNH}_{4}^{+}, 50\right), 364.2\left(\mathrm{MNa}^{+}, 100\right)$ $405.2\left(\mathrm{MNa}^{+} \mathrm{MeCN}, 95 \%\right)$. HRMS $\left(\mathrm{ES}^{+}\right)$Calcd. for $\mathrm{C}_{18} \mathrm{H}_{26} \mathrm{~N}_{2} \mathrm{NaO}_{5} \mathrm{~S}\left(\mathrm{MNa}^{+} \mathrm{MeCN}\right)$ 405.1460. Found 405.1466. 


\section{(4RS,1'SR)-4-(1'-Hydroxypropyl)-[1,3]oxazinan-2-one 17}

$\mathrm{N}$-Sulfonyloxy carbamate $16(273 \mathrm{mg}, 0.80 \mathrm{mmol})$ was subjected to the general aminohydroxylation procedure. The crude material was purified by flash column chromatography (ethyl acetate) to furnish the title compound $(92.9 \mathrm{mg}, 73 \%$ ) as a white solid; m.p. $102-104{ }^{\circ} \mathrm{C} ; v_{\max }\left(\mathrm{KBr}\right.$ disk) 3331, 3241, 1729, $1656 \mathrm{~cm}^{-1} ; \delta_{\mathbf{H}}$ $\left(400 \mathrm{MHz} ; \mathrm{CDCl}_{3}\right) 6.80(1 \mathrm{H}, \mathrm{br}), 4.37(1 \mathrm{H}, \mathrm{dt}, J 10.8,4.0), 4.19(1 \mathrm{H}, \mathrm{td}, J 11.0,2.8)$, $3.64(1 \mathrm{H}, \mathrm{br}), 3.58(1 \mathrm{H}, \mathrm{ddd}, J 7.6,5.2,3.6), 3.54-3.49(1 \mathrm{H}, \mathrm{m}, 2.06-1.96(1 \mathrm{H}, \mathrm{m})$, $1.82-1.78(1 \mathrm{H}, \mathrm{m}), 1.52-1.40(2 \mathrm{H}, \mathrm{m}), 0.98\left(3 \mathrm{H}, \mathrm{t}, J\right.$ 7.5, $\left.\mathrm{CH}_{3}\right) ; \delta_{\mathrm{C}}(100.6 \mathrm{MHz}$ $\left.\mathrm{CDCl}_{3}\right)$ 156.2, 73.2, 65.9, 55.2, 25.1, 20.6, 10.6; $\mathrm{m} / \mathrm{z}(\mathrm{CI}) 177\left(\mathrm{MNH}_{4}^{+}, 5\right), 160$ $\left(\mathrm{M}+\mathrm{H}^{+}, 100 \%\right)$; HRMS (CI) Calcd. for $\mathrm{C}_{7} \mathrm{H}_{14} \mathrm{NO}_{3}\left(\mathrm{MH}^{+}\right)$160.0974. Found 160.0972.

tert-Butyl 2-(mesitylsulfonyloxycarbamoyloxy)methyl-2H-pyrrole-1 (5H)carboxylate 18

Alcohol (498 mg, $2.50 \mathrm{mmol}$ ) was subjected to the general $N$-hydroxy carbamate formation procedure and purified by flash column chromatography (petrol:ethyl acetate, 2:1) to afford the corresponding $\mathrm{N}$-hydroxy carbamate $(323 \mathrm{mg}, 50 \%)$. The $\mathrm{N}$-hydroxy carbamate $(281 \mathrm{mg}, 1.09 \mathrm{mmol})$ was then subjected to the general mesitylation procedure and purified by flash column chromatography (petrol:ethyl acetate, 4:1) to afford $\mathrm{N}$-sulfonyloxy carbamate $\mathbf{1 8}(290 \mathrm{mg}, 60 \%)$ as a clear oil; $\mathrm{v}_{\max }$ (thin film) $3158,1774,1371,1193 \mathrm{~cm}^{-1} ; \delta_{\mathbf{H}}\left(400 \mathrm{MHz}, \mathrm{CDCl}_{3}\right.$ ) (mixture of BOC rotamers) 8.37, $8.20(1 \mathrm{H}, \mathrm{s}), 6.98(2 \mathrm{H}, \mathrm{s}), 5.79(1 \mathrm{H}, \mathrm{ddd}, J 13.9,5.0,1.2), 5.51(1 \mathrm{H}$, dd, $J 13.9,1.3), 4.64,4.52(1 \mathrm{H}, \mathrm{m}), 4.32(1 \mathrm{H}, \mathrm{dd}, J 4.2,1.3), 4.20-4.14(2 \mathrm{H}, \mathrm{m}), 4.13-$ $4.09(1 \mathrm{H}, \mathrm{m}), 2.66(6 \mathrm{H}, \mathrm{s}), 2.32(3 \mathrm{H}, \mathrm{s}), 1.47,1.46(9 \mathrm{H}, \mathrm{s}) ; \delta_{\mathrm{C}}\left(100.6 \mathrm{MHz}, \mathrm{CDCl}_{3}\right)$ (mixture of BOC rotamers) 155.6, 154.2, 153.8, 144.5, 141.9, 131.7, 128.2, 127.7, $126.3,126.2,80.4,80.0,66.3,65.9,63.2,63.0,53.7,53.5,28.4,22.9,21.2 ; \boldsymbol{m} / z\left(\mathrm{ES}^{+}\right)$ 
$499.3\left(\mathrm{MNH}_{4}{ }^{+} \mathrm{MeCN}, 100 \%\right)$. HRMS $\left(\mathrm{ES}^{+}\right)$Calcd. for $\mathrm{C}_{20} \mathrm{H}_{28} \mathrm{~N}_{2} \mathrm{NaO}_{7} \mathrm{~S}\left(\mathrm{MNa}^{+}\right)$ 463.1515. Found 463.1520.

\section{4-Hydroxymethyl-2-oxo-hexahydro-pyrrole-3,4-oxazole-5-carboxylic acid tert-} butyl ester 19

$\mathrm{N}$-Sulfonyloxy carbamate $18(115 \mathrm{mg}, 0.26 \mathrm{mmol})$ was subjected to the general aminohydroxylation procedure. The crude material was purified by flash column chromatography (ethyl acetate) to furnish the title compound $(42.2 \mathrm{mg}, 63 \%)$ as a pale

yellow solid; m.p. $117-118^{\circ} \mathrm{C} ; v_{\max }\left(\mathrm{CHCl}_{3}\right) 3333,1750,1690 \mathrm{~cm}^{-1} ; \delta_{\mathbf{H}}(400 \mathrm{MHz}$, $\left.\mathrm{CDCl}_{3}\right) 6.58(1 \mathrm{H}, \mathrm{br}), 5.10-5.05(1 \mathrm{H}, \mathrm{m}), 4.52(1 \mathrm{H}, \mathrm{t}, J$ 7.4), $4.02(1 \mathrm{H}, \mathrm{dd}, J$ 11.0, 5.2), $3.89(1 \mathrm{H}, \mathrm{m}), 3.80(1 \mathrm{H}, \mathrm{m}), 3,76(1 \mathrm{H}, \mathrm{dd}, J 11.1,6.6), 3.67(1 \mathrm{H}, \mathrm{m}), 2.20(1 \mathrm{H}$, br), $1.46(9 \mathrm{H}, \mathrm{s}) ; \delta_{\mathrm{C}}\left(100.6 \mathrm{MHz}, \mathrm{CDCl}_{3}\right) 159.3,154.9,81.3,77.6,60.8,57.8,52.5$, 28.4, 18.5; m/z (FI) 258.1 $\left(\mathrm{M}^{+}, 100 \%\right)$. HRMS (FI) Calcd. for $\mathrm{C}_{11} \mathrm{H}_{18} \mathrm{~N}_{2} \mathrm{O}_{5}\left(\mathrm{M}^{+}\right)$ 258.1216. Found 258.1223.

\section{(2-Benzyl-2,5-dihydrofuran-2-yl)methyl mesitylsulfonyloxy carbamate 20}

Alcohol (204 mg, $1.00 \mathrm{mmol}$ ) was subjected to the general $N$-hydroxy carbamate formation procedure and purified by flash column chromatography (petol:ether, 1:1) to afford the corresponding N-hydroxy carbamate (191 mg, 73\%). The N-hydroxy carbamate $(129 \mathrm{mg}, 0.49 \mathrm{mmol})$ was then subjected to the general mesitylation procedure and purified by flash column chromatography (petrol:ethyl acetate, $9: 1 \rightarrow 5: 1)$ to afford $\mathrm{N}$-sulfonyloxy carbamate $20(117 \mathrm{mg}, 54 \%)$ as a clear oil; $v_{\max }$ (thin film) $3268,1774,1372,1193 \mathrm{~cm}^{-1} ; \delta_{\mathbf{H}}\left(400 \mathrm{MHz}, \mathrm{CDCl}_{3}\right) 8.42(1 \mathrm{H}, \mathrm{s}), 7.26-$ $7.22(3 \mathrm{H}, \mathrm{m}), 7.14-7.10(2 \mathrm{H}, \mathrm{m}), 6.98(2 \mathrm{H}, \mathrm{s}), 5.77(1 \mathrm{H}, \mathrm{dt}, J 6.1,1.5), 5.49(1 \mathrm{H}, \mathrm{dt}$, $J 6.1,1.4), 4.51(1 \mathrm{H}, \mathrm{dt}, J 12.9,1.7), 4.31(1 \mathrm{H}, \mathrm{dt}, J 13.0,1.8), 4.13,4.04(2 \mathrm{H}, \mathrm{ABq}$ 
$J$ 11.1), 2.83, $2.74(2 \mathrm{H}, \mathrm{ABq}, J 13.7), 2.69(6 \mathrm{H}, \mathrm{s}), 2.29(3 \mathrm{H}, \mathrm{s}) ; \delta_{\mathrm{C}}(125.7 \mathrm{MHz}$ $\left.\mathrm{CDCl}_{3}\right)$ 155.5, 144.6, 139.8, 136.2, 132.7, 131.9, 131.8, 129.3, 128.2, 128.0, 126.6, 91.5, 75.6, 69.8, 42.0, 23.1, 19.5; $\boldsymbol{m} / z\left(\mathrm{ES}^{+}\right) 454.2\left(\mathrm{MNa}^{+}, 15\right), 490.3\left(\mathrm{MNH}_{4}{ }^{+} \mathrm{MeCN}\right.$, 100\%). HRMS $\left(\mathrm{ES}^{+}\right)$Calcd. for $\mathrm{C}_{22} \mathrm{H}_{25} \mathrm{NNaO}_{6} \mathrm{~S}\left(\mathrm{MNa}^{+}\right)$454.1295. Found 454.1288.

\section{Acetic acid 4-amino-5-benzyl-5-hydroxymethyl-tetrahydrofuran-3-yl ester 21}

$N$-Sulfonyloxy carbamate $20(57.0 \mathrm{mg}, 0.13 \mathrm{mmol})$ was subjected to the general aminohydroxylation procedure. The crude material was purified by flash column chromatography (petrol:ethyl acetate, 1:2) to furnish the title compound (22.6 mg, $70 \%$ ) as a white solid; m.p. $167-168^{\circ} \mathrm{C} ; v_{\max }\left(\mathrm{KBr}\right.$ disk) $3465,3266,1727 \mathrm{~cm}^{-1} ; \delta_{\mathbf{H}}$ $\left(400 \mathrm{MHz}, \mathrm{CDCl}_{3}\right)$ 7.33-7.21 (5H, m), $6.45(1 \mathrm{H}, \mathrm{s}), 5.15$ (1H, ddd, J 7.6, 4.4, 1.5), $4.24(1 \mathrm{H}, \mathrm{d}, J$ 7.5), 4.19 (1H, dd, $J 13.0,1.4), 4.11(1 \mathrm{H}, \mathrm{dd}, J 11.6,4.4), 3.57$ (2H, s), 2.86, $2.81(2 \mathrm{H}, \mathrm{ABq}, J 14.0), 1.90-1.50(1 \mathrm{H}, \mathrm{br}) ; \delta_{\mathrm{C}}\left(100.6 \mathrm{MHz}, \mathrm{CDCl}_{3}\right) 165.8$ $134.9,130.3,128.5,128.1,127.3,78.7,72.2,71.4,59.0,41.5 ; m / z\left(\mathrm{ES}^{+}\right) 250\left(\mathrm{MH}^{+}\right.$ 66), $291\left(\mathrm{MHMeCN}^{+}, 100 \%\right)$. HRMS (ES $\left.{ }^{+}\right)$Calcd. for $\mathrm{C}_{13} \mathrm{H}_{16} \mathrm{NO}_{4}\left(\mathrm{MH}^{+}\right)$250.1079. Found 250.1079.

\section{References:}

1. Greck, C.; Bischoff, L.; Ferreira, F.; Genet, J. P., J. Org. Chem. 1995, 60, 7010.

2. Donohoe, T. J.; Helliwell, M.; Johnson, P. D.; Keenan, M. Chem. Commun. 2001, 2078.

3. Donohoe, T. J.; Cowley, A.; Johnson, P. D.; Keenan, M. J. Am. Chem. Soc. 2002, $124,12934$.

4. Donohoe, T. J.; Johnson, P. D.; Keenan, M.; Pye, R. J. Organic Lett. 2004, 6, 2583. 


\section{$O$-2-Mesitylenesulfonyl $N$-hydroxy $(E)$-hex-2-enyl carbamate 3}
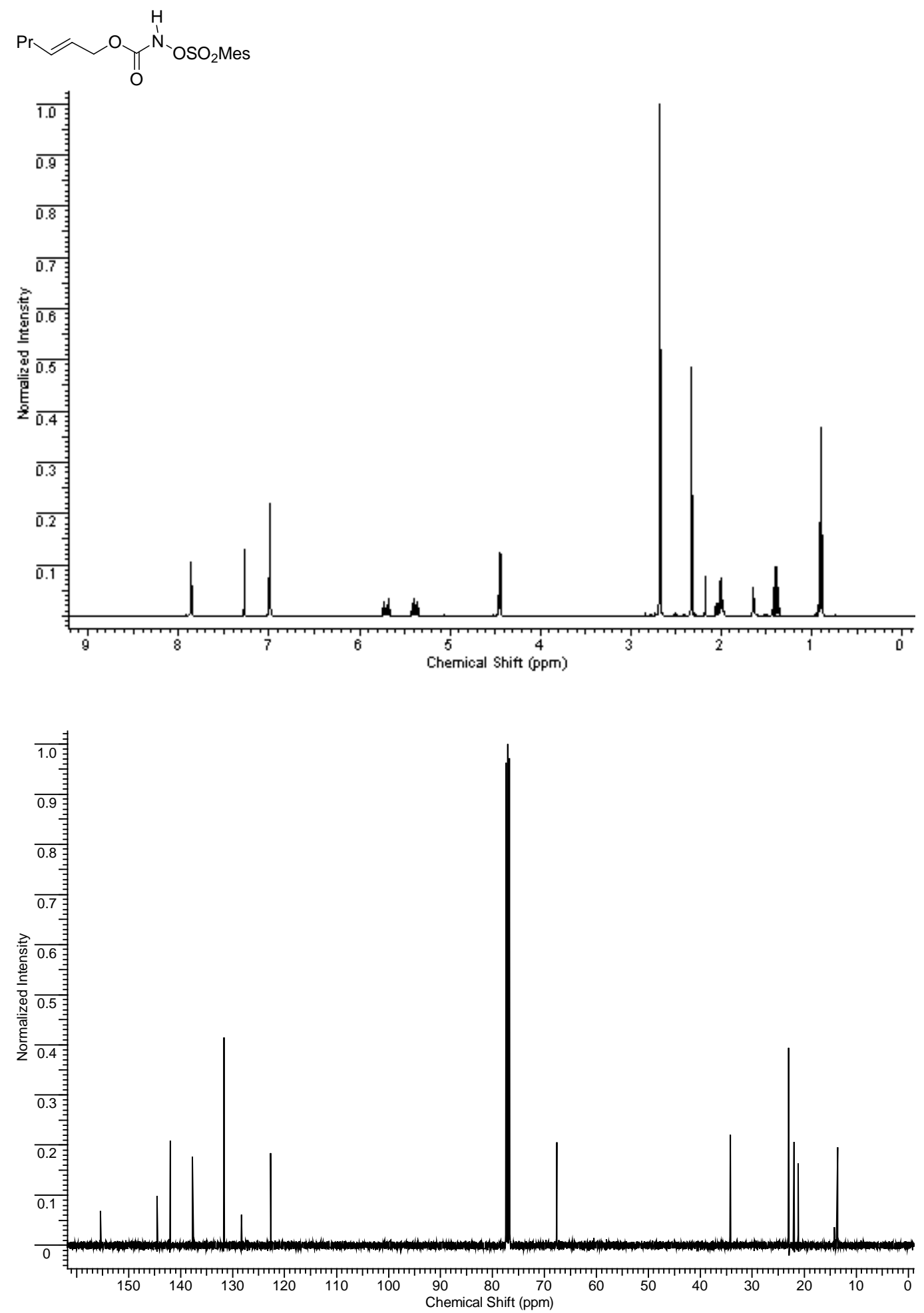
$O$-2-Mesitylenesulfonyl $N$-hydroxy $(Z)$-hex-2-enyl carbamate 5
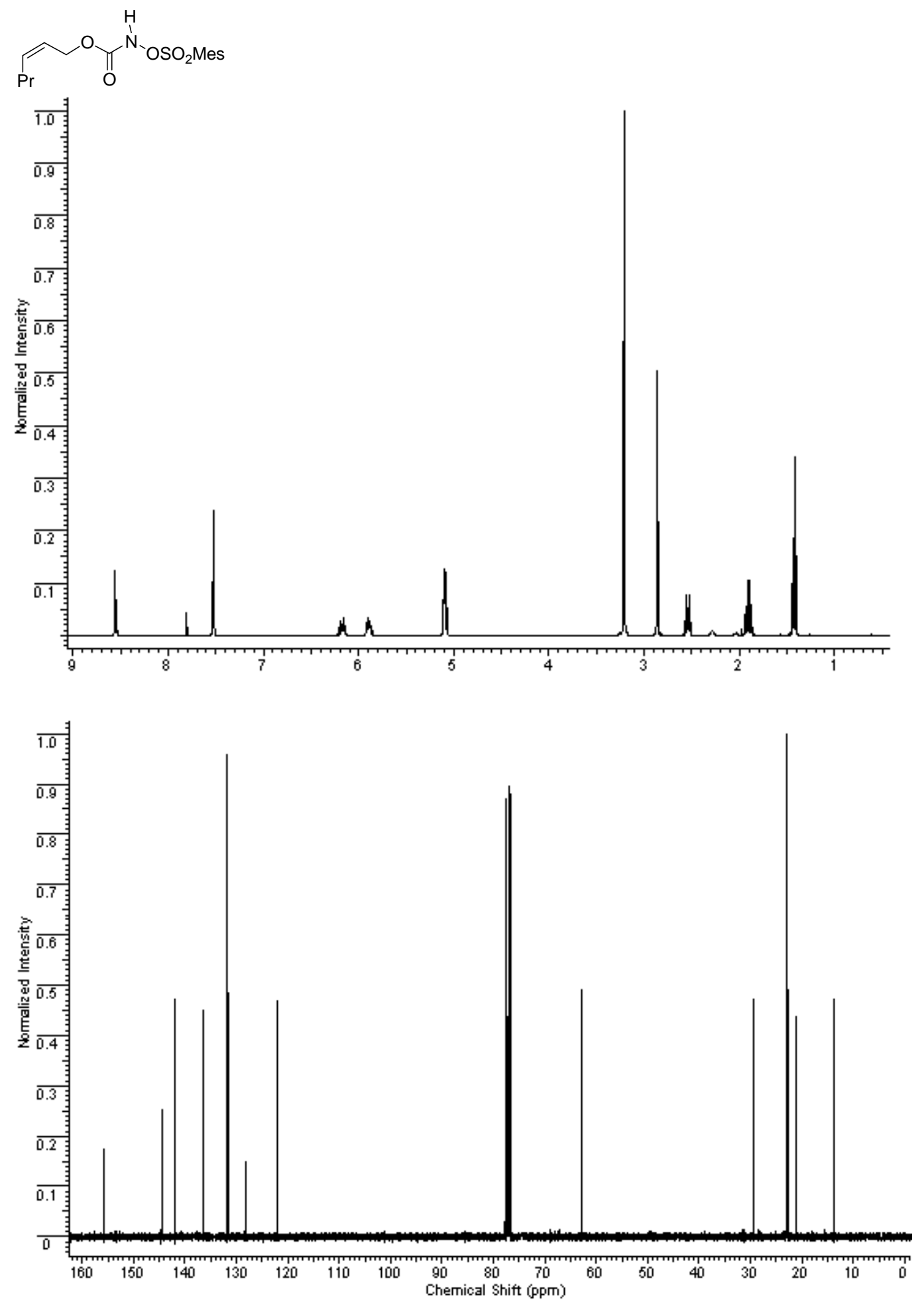


\section{$O$-2-Mesitylenesulfonyl $N$-hydroxy cyclohex-2-enyl carbamate 7}
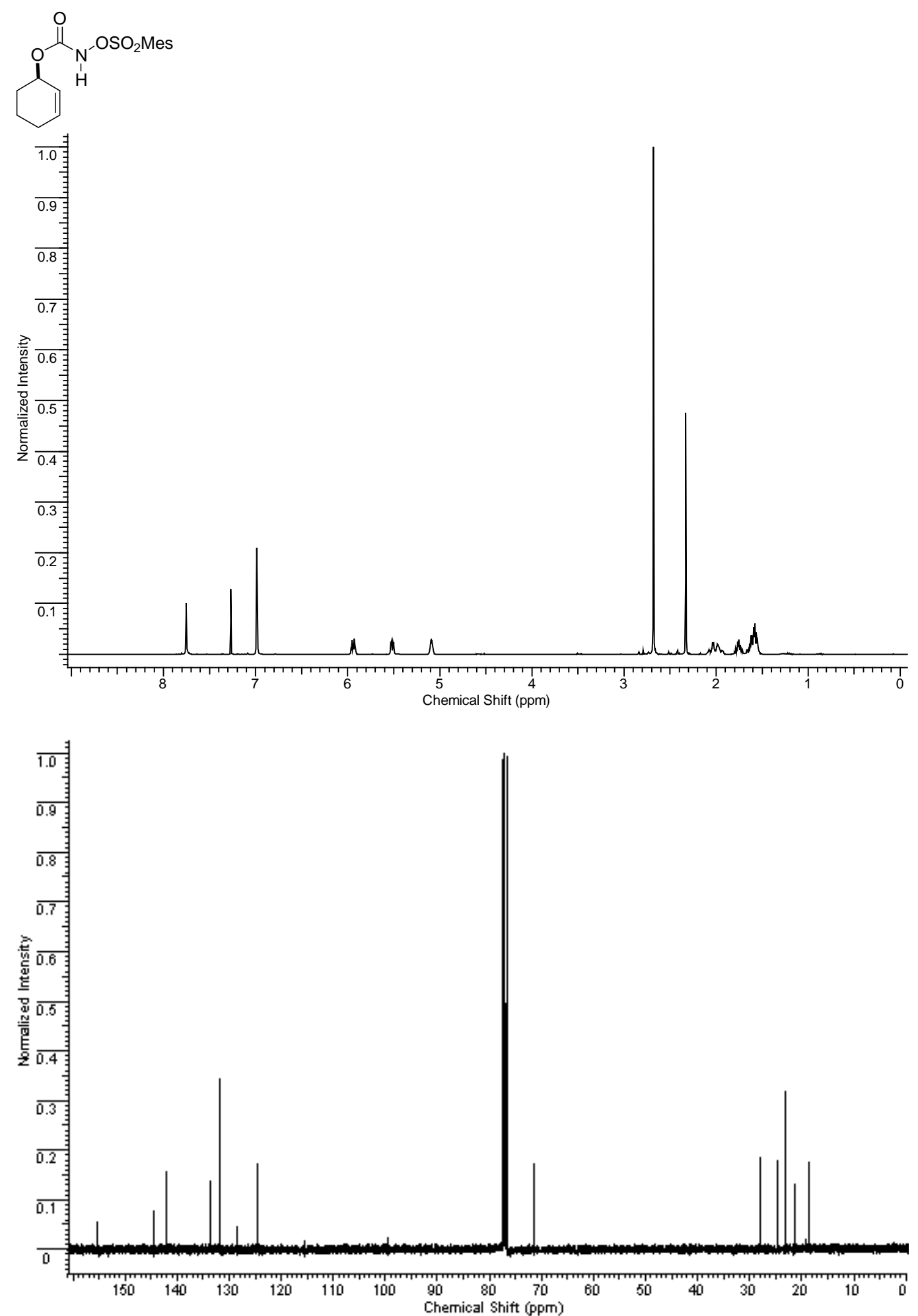


\section{$O$-2-Mesitylenesulfonyl $N$-hydroxy 1-cyclohexene-1-yl-pentyl carbamate 9}
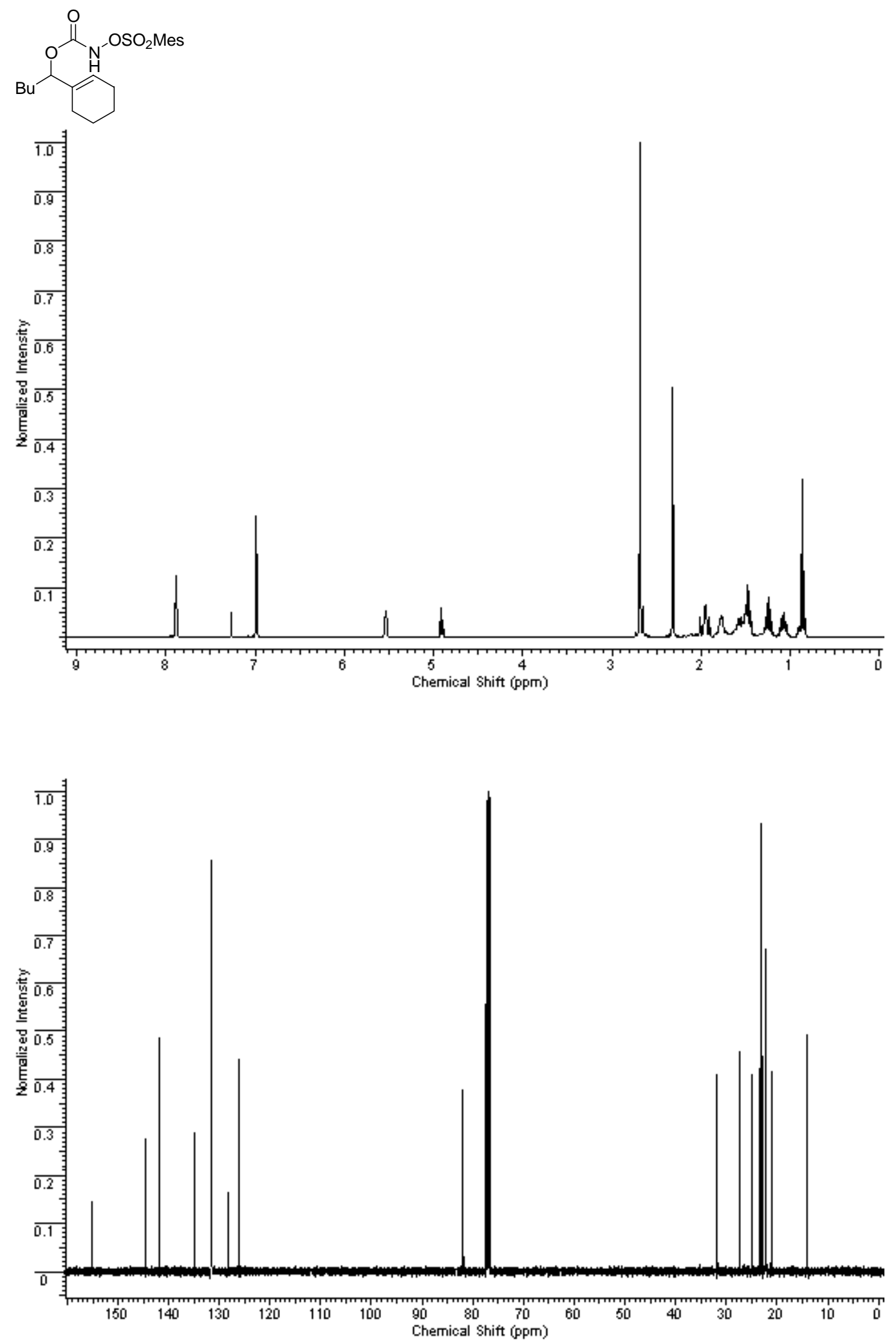


\section{$O$-2-Mesitylenesulfonyl $N$-hydroxy $(E)$-hex-3-enyl carbamate 11}
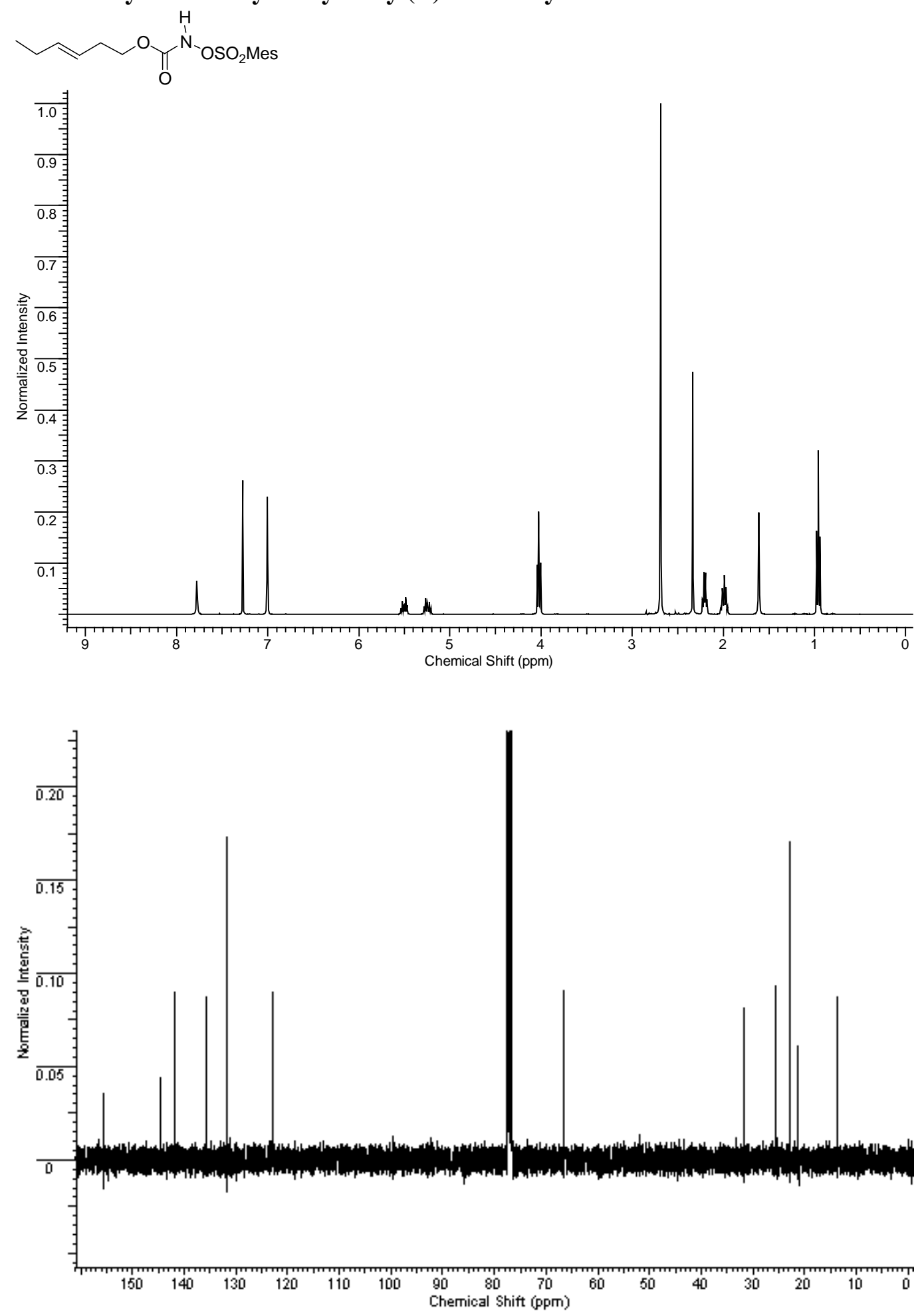
(4RS,1'RS)-4-(1'-Hydroxypropyl)-[1,3]oxazinan-2-one 12
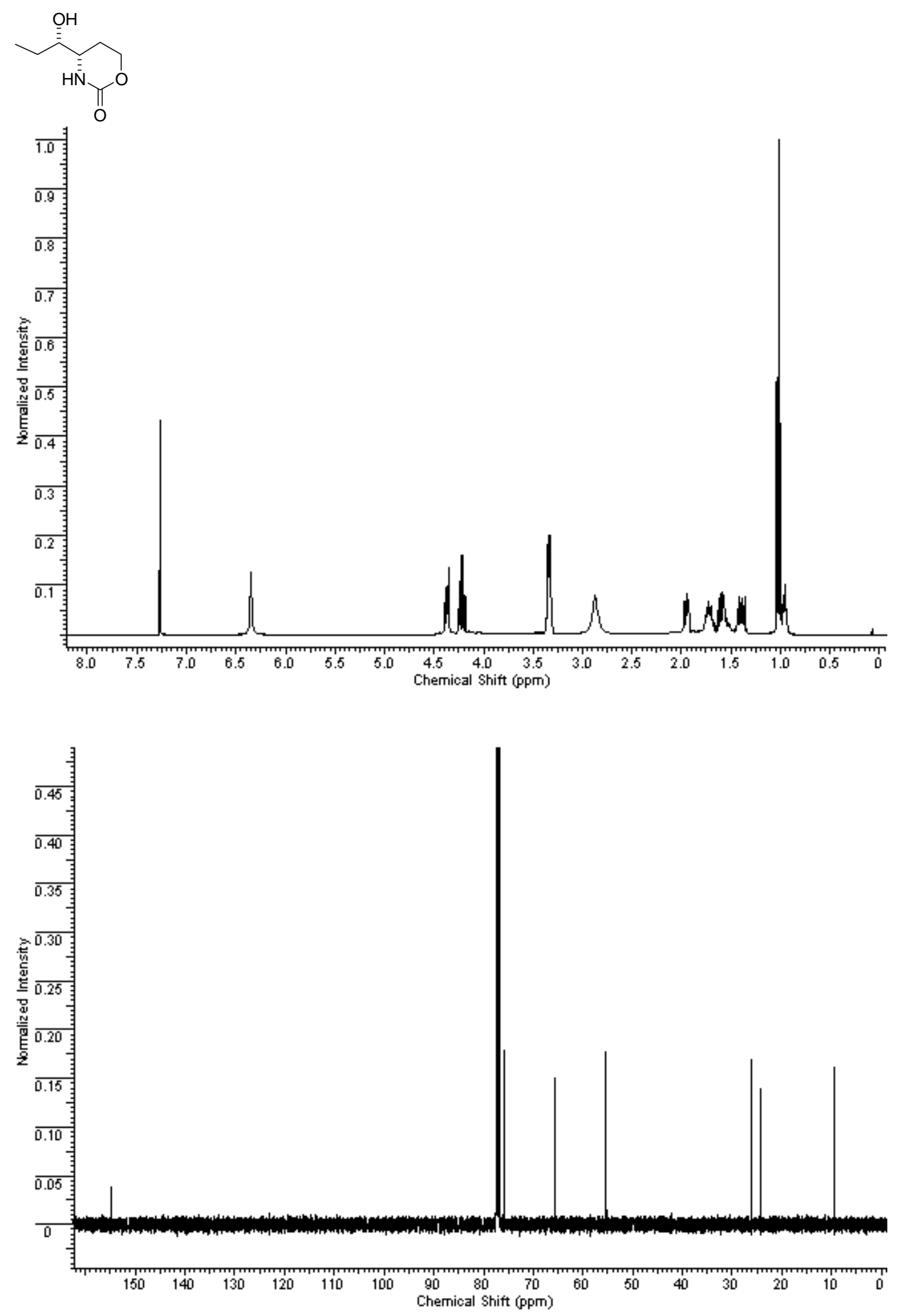
Osmate ester 13
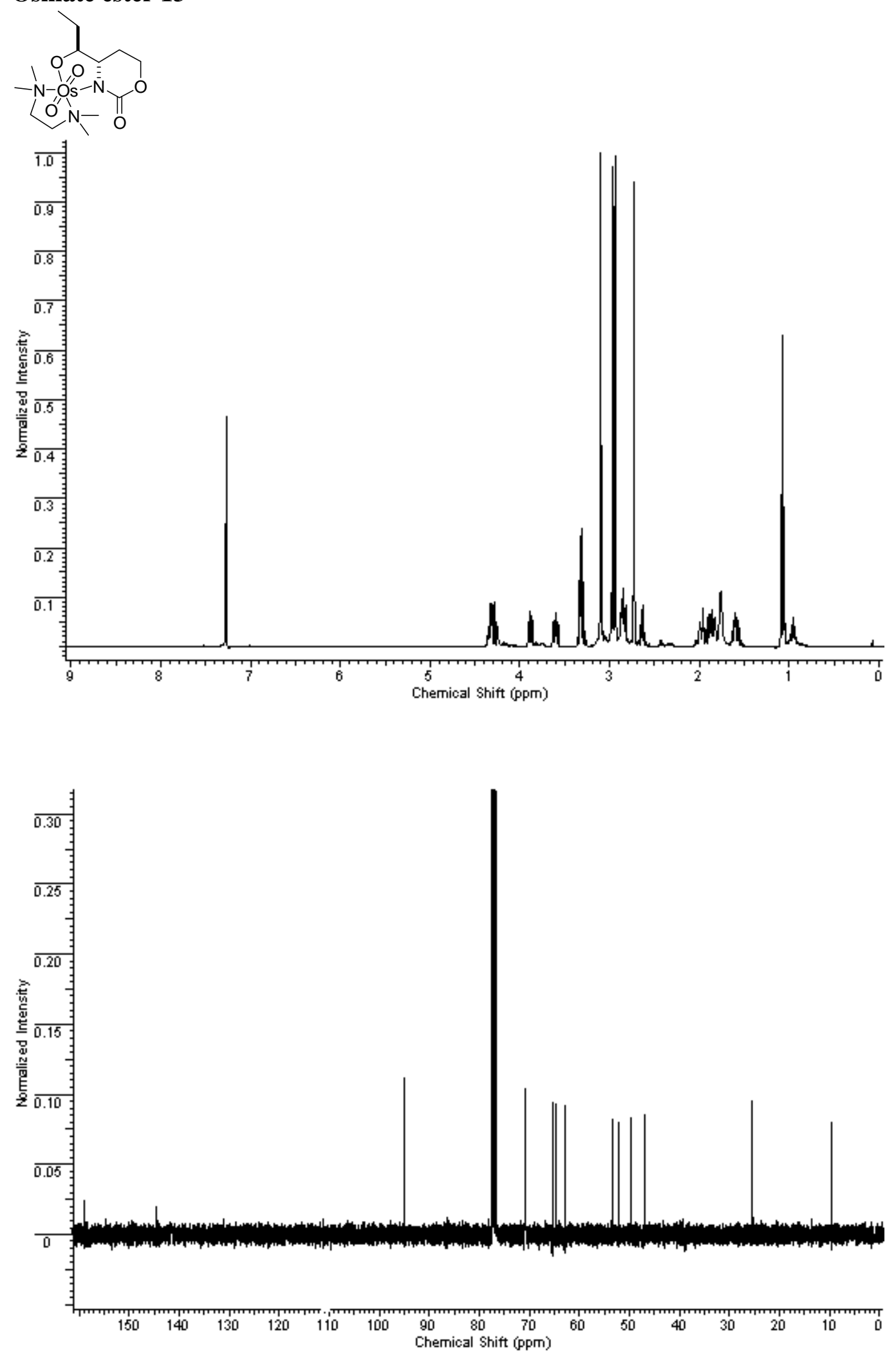


\section{$O$-2-Mesitylenesulfonyl $N$-hydroxy but-3-enyl carbamate 14}<smiles>C=CCCOC(=O)NOS(=O)(=O)c1c(C)cc(C)cc1C</smiles>
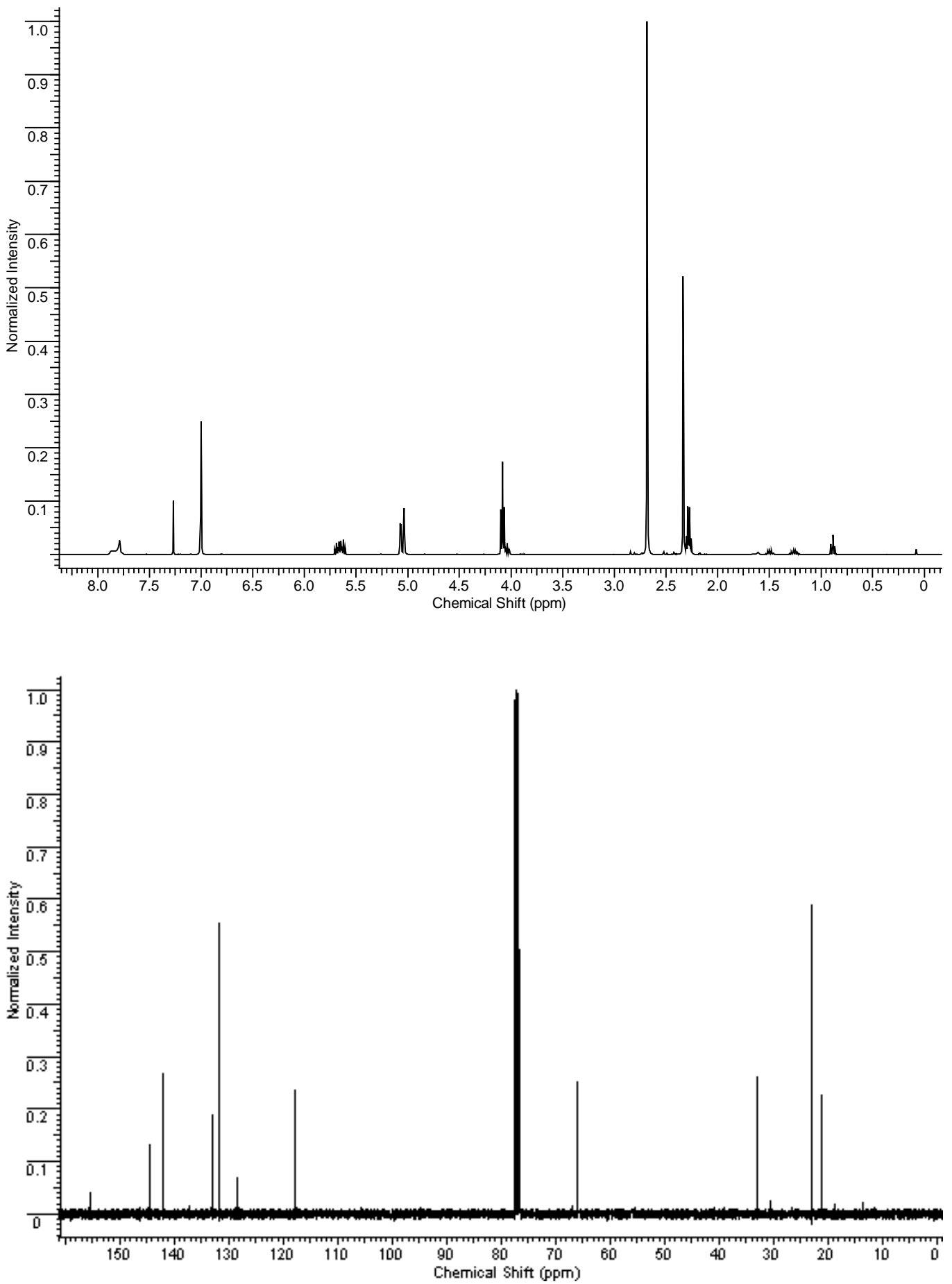
$O$-2-Mesitylenesulfonyl $N$-hydroxy $(Z)$-hex-3-enyl carbamate 16
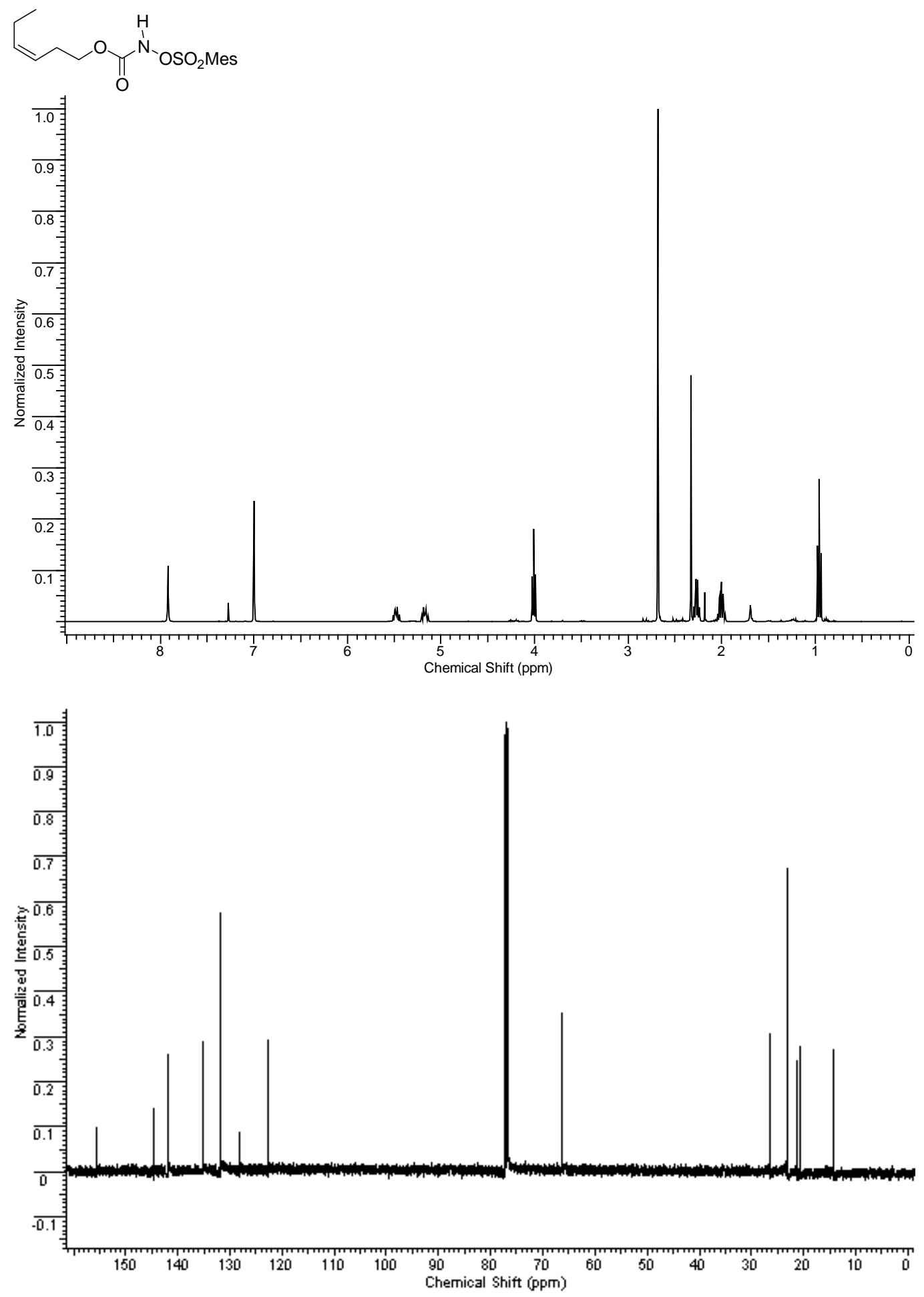
(4RS,1'SR)-4-(1'-Hydroxypropyl)-[1,3]oxazinan-2-one 17<smiles>CC[C@H](O)[C@H]1CCOC(=O)N1</smiles>
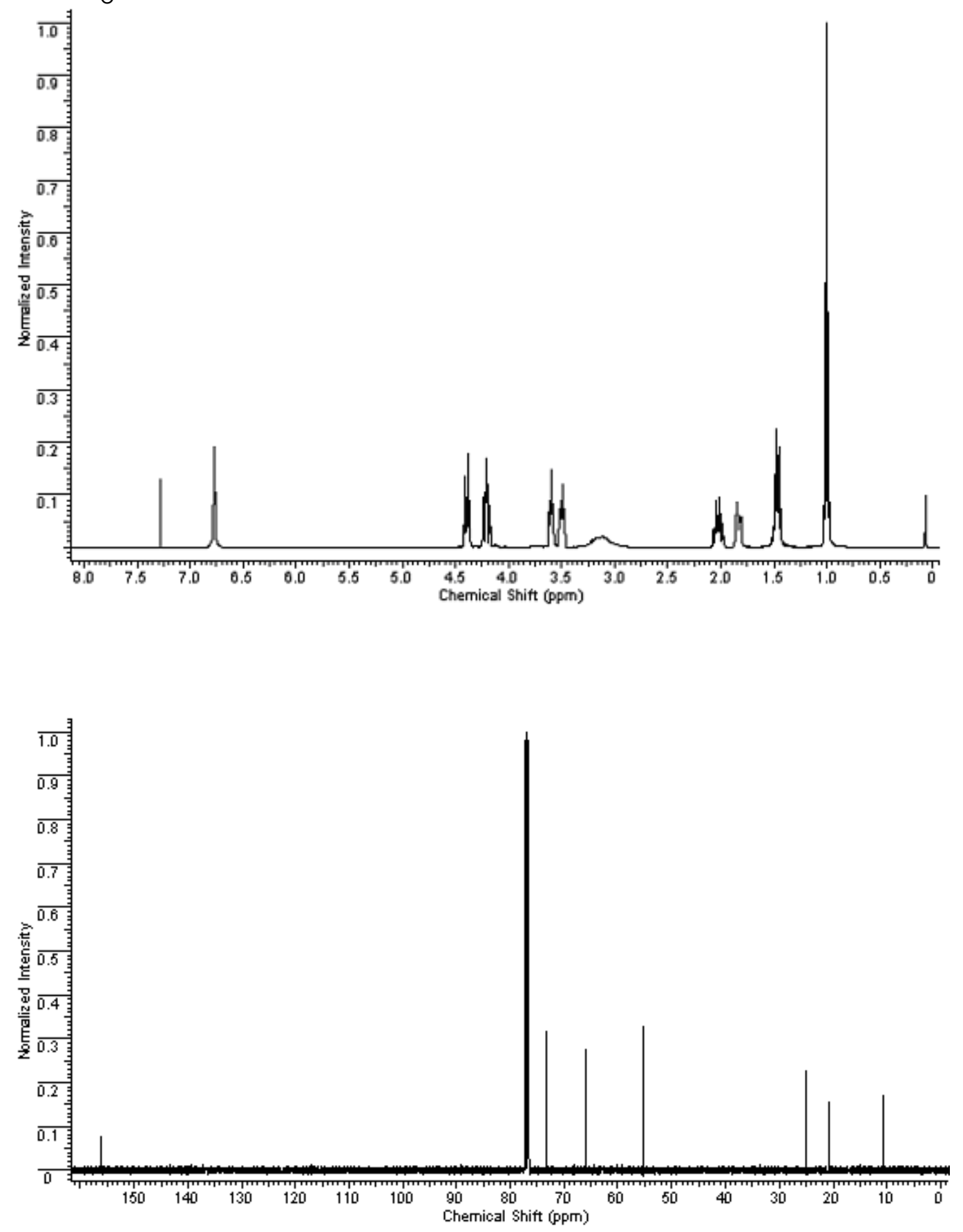
tert-Butyl 2-((mesutylsulfonyloxycarbamoyloxy)methyl-2H-pyrrole-1 (5H)carboxylate 18
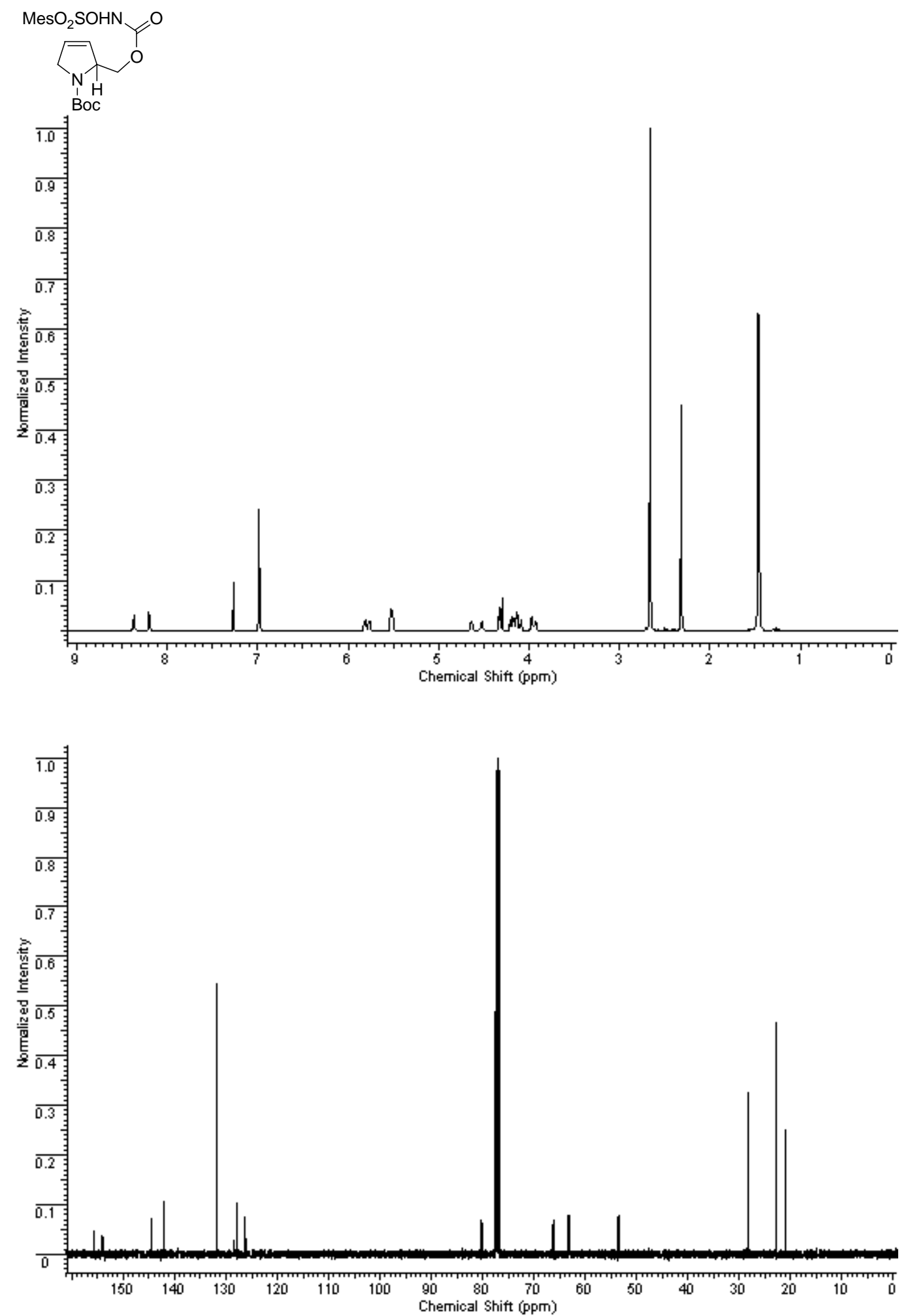
4-Hydroxymethyl-2-oxo-hexahydro-pyrrole-3,4-oxazole-5-carboxylic acid tertbutyl ester 19
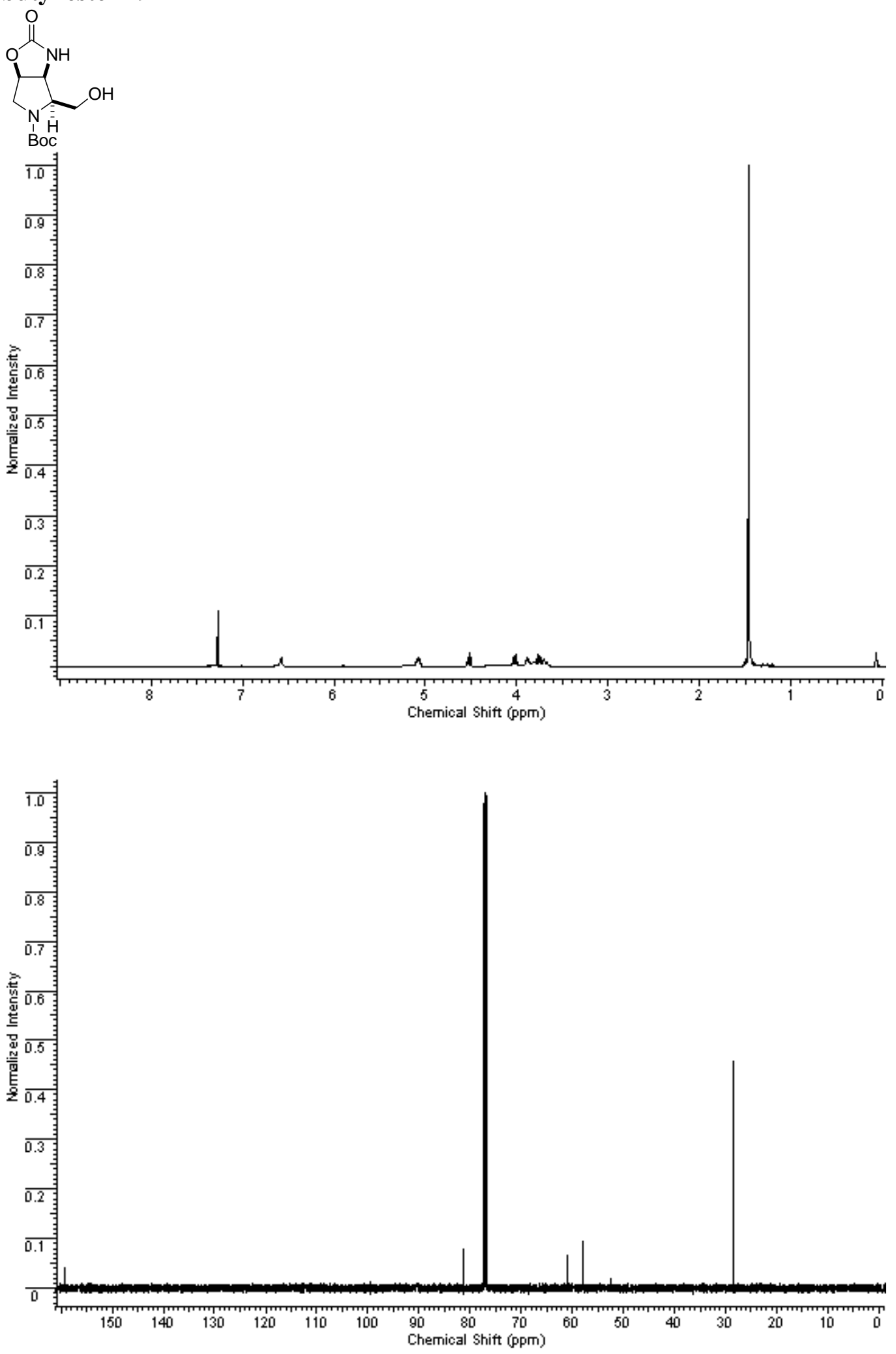


\section{(2-Benzyl-2,5-dihydrofuran-2-yl)methyl mesitylsulfonyloxy carbamate 20}<smiles>COC(OC)OC(=O)NC(=O)OCC1(Cc2ccccc2)C=CCO1</smiles>
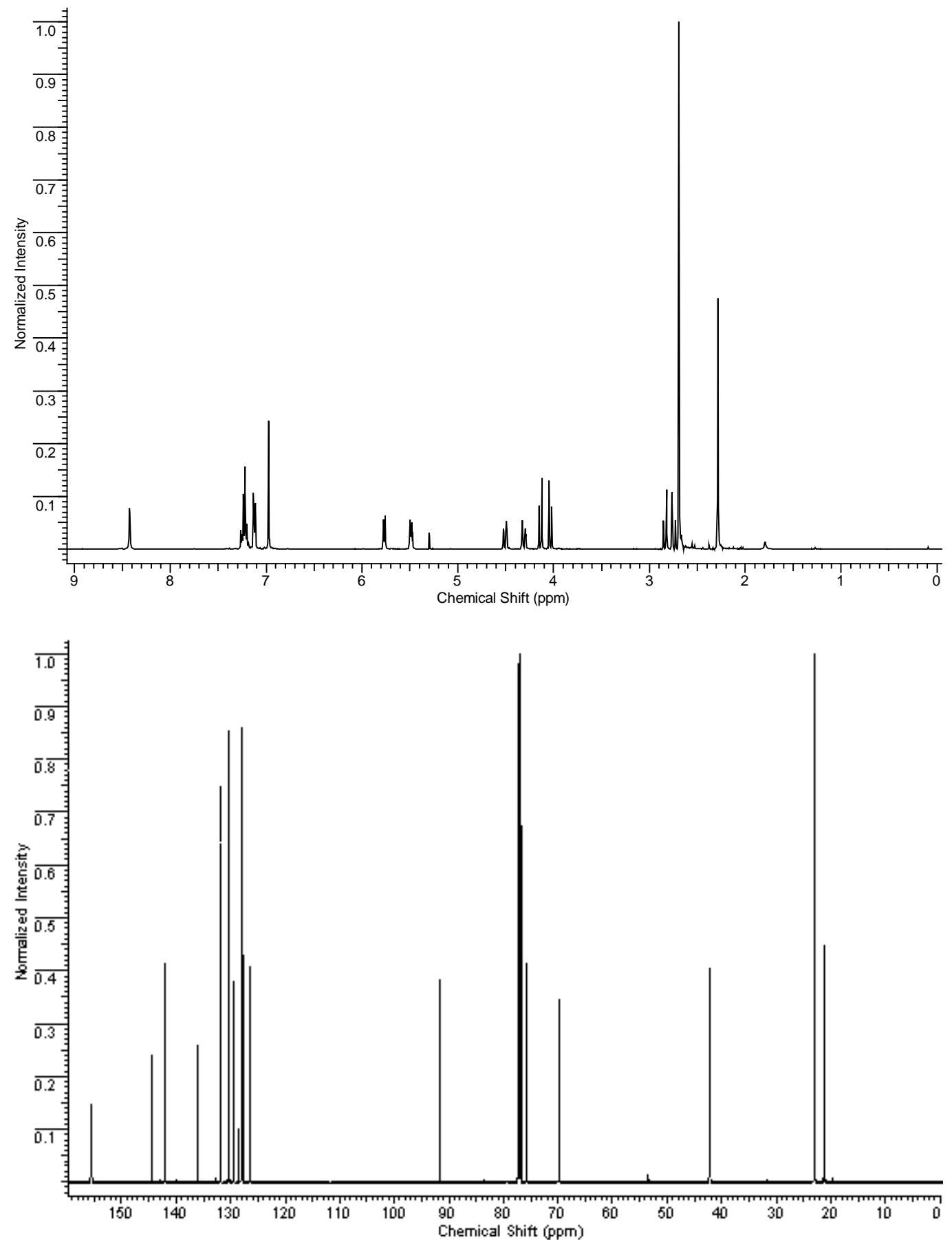
Acetic acid 4-amino-5-benzyl-5-hydroxymethyl-tetrahydrofuran-3-yl ester 21
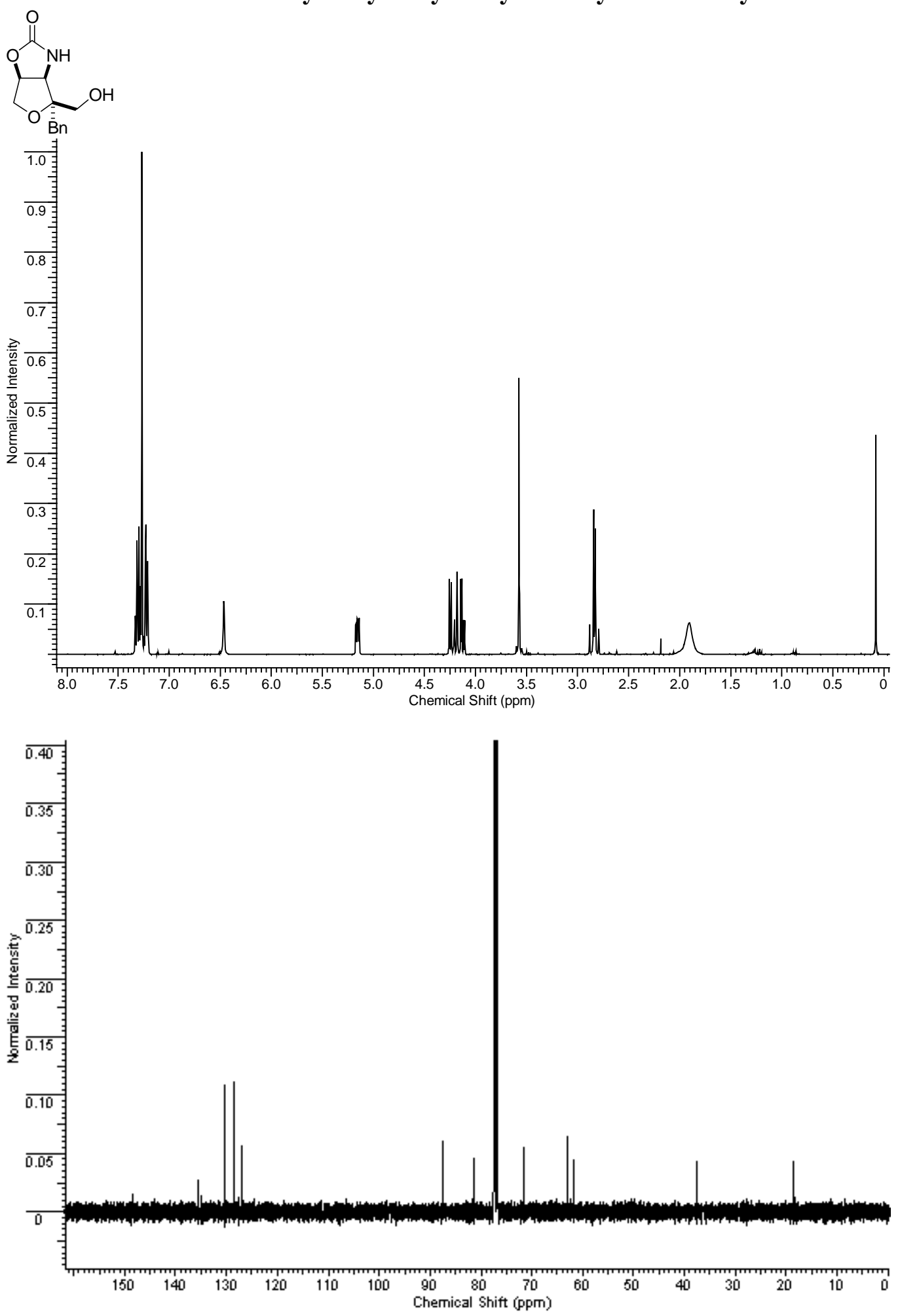